\title{
Electron-nuclei spin dynamics in II-VI semiconductor quantum dots
}

\author{
C. Le Gall, A. Brunetti, H. Boukari, and L. Besombes* \\ CEA-CNRS group “Nanophysique et semiconducteurs," Institut Néel, CNRS \& Université Joseph Fourier, BP 166, \\ F-38042 Grenoble Cedex 9, France
}

(Received 6 April 2012; published 10 May 2012)

\begin{abstract}
We report on the dynamics of optically induced nuclear spin polarization in individual CdTe/ZnTe quantum dots loaded with one electron by modulation doping. The fine structure of the hot trion (charged exciton $X^{-}$ with an electron in the $P$ shell) is identified in photoluminescence excitation spectra. A negative polarization rate of the photoluminescence, optical pumping of the resident electron, and the built up of dynamic nuclear spin polarization (DNSP) are observed in time-resolved optical pumping experiments when the quantum dot is excited at higher energy than the hot trion triplet state. The time and magnetic field dependence of the polarization rate of the $X^{-}$emission allows us to probe the dynamics of formation of the DNSP in the optical pumping regime. We demonstrate using time-resolved measurements that the creation of a DNSP at $B=0 \mathrm{~T}$ efficiently prevents longitudinal spin relaxation of the electron caused by fluctuations of the nuclear spin bath. The DNSP is built in the microsecond range at high excitation intensity. A relaxation time of the DNSP in about $10 \mu \mathrm{m}$ is observed at $B=0 \mathrm{~T}$ and significantly increases under a magnetic field of a few milli-Tesla. We discuss mechanisms responsible for the fast initialization and relaxation of the diluted nuclear spins in this system.
\end{abstract}

DOI: 10.1103/PhysRevB.85.195312

PACS number(s): 72.25.Fe, 78.55.Et

\section{INTRODUCTION}

The confinement of single electrons in semiconductor quantum dots (QDs) and the control of their spin has been motivated by perspectives of using the electron spin as the ultimate solid state system to store and process quantum information. In the commonly studied III-V semiconductor QDs, the hyperfine interaction of the electron with the fluctuating nuclear spins limits the time scale on which an electron spin can be manipulated at low magnetic field. It has been proposed that a full polarization of the nuclei could cancel the decoherence of the electron induced by the fluctuating hyperfine field. ${ }^{1}$ Alternatively, the decoherence created by nuclei could be circumvented by using isotopically purified II-VI materials ${ }^{2}$ since $\mathrm{Zn}, \mathrm{Cd}, \mathrm{Mg}, \mathrm{O}$, Se, and $\mathrm{Te}$ all have dominant isotopes without nuclear spins. However, as highlighted in Refs. 3 and 4, the interaction between a confined electron in a II-VI QD and the low density of nuclear spins $I=1 / 2$ in a QD volume 10 to 100 times smaller than InAs/GaAs QDs leads to some spin dynamics which is fundamentally different from the one observed in III-V systems. ${ }^{3,4}$

Due to the small QD size and low density of nuclear spins, the electron-nuclei dynamics in II-VI QDs is ruled by a large Knight field and significant nuclear spin fluctuations despite a small Overhauser field. Consequently, the nucleiinduced spin decoherence of the electron is also an issue in II-VI QDs. However, the builtup of a dynamic nuclear spin polarization (DNSP) at $B=0 \mathrm{~T}$ can be much faster than the relaxation induced by the dipole interaction between nuclear spins, allowing the creation of a strong nonequilibrium DNSP. ${ }^{3}$ Under these conditions, decoherence of the electron should be efficiently suppressed. Experimental study of the electron-nuclear dynamics in II-VI QDs are few. Providing a thorough experimental study of this system at a single dot level is the aim of this work.

In this paper we report on the dynamics of coupled electron and nuclear spins polarization in individual CdTe/ZnTe QDs with a resident electron introduced by aluminum modulation doping. Here, in contrast to gated structures where the number of resident charges is controlled by an applied voltage, we use the characteristic spectral feature of the charged exciton triplet state observed in photoluminescence excitation spectra (PLE) to identify QDs containing a single resident electron. Nonresonant circularly polarized excitation of the negatively charged exciton has been shown to lead to a polarization of the nuclear spins in both III-V ${ }^{5}$ and II-VI QDs ${ }^{4}$ and will be used throughout this study to build up and probe DNSP. In II-VI QDs, the Overhauser shift is much smaller than the photoluminescence (PL) linewidth and cannot be observed directly: The nuclear field is detected through the polarization rate of the resident electron which is controlled by the nuclear spin fluctuations (NSF).

We give a description of the studied structures and experimental techniques in Sec. II of this paper. In Sec. III we discuss nuclear spin polarization in II-VI QDs, and derive orders of magnitude for the effective hyperfine fields encountered in this system. Experimental evidences of $X^{-}$triplet states and details about the mechanism of optical spin injection and build up of negative circular polarization in singly charged $\mathrm{CdTe} / \mathrm{ZnTe}$ QDs are presented in Sec. IV. In Sec. V we describe how the polarization of nuclear spins influences spin dynamics of the confined electron. The dynamics of nuclear spin polarization is considered in Sec. VI. Finally, in Sec. VII we present and discuss results of coupled electron-nuclei spin decay in the absence of optical excitation.

\section{SAMPLE AND EXPERIMENT}

The sample used in this study is grown on a ZnTe substrate and contains CdTe/ZnTe QDs. A 6.5-monolayer-thick CdTe layer is deposited at $280^{\circ} \mathrm{C}$ by atomic layer epitaxy on a $\mathrm{ZnTe}$ barrier grown by molecular beam epitaxy at $360^{\circ} \mathrm{C}$. The dots are formed by the high tellurium deposition process described 
in Ref. 7 and protected by a 100-nm-thick ZnTe top barrier. ${ }^{6} \mathrm{~A}$ 20-nm-thick Al doped ZnTe layer is introduced $30 \mathrm{~nm}$ above the QDs leading to an average negative charging of the QDs. The height of the QDs core is about 2-3 nanometers and their diameter is 10 to $20 \mathrm{~nm}$. We estimate an average QD volume of about $250 \mathrm{~nm}^{3}$ containing $\approx 8000$ nuclei, 1200 of which carry a spin $1 / 2$.

Optical addressing of individual QDs containing a single electron is achieved using microspectroscopy techniques. A high refractive index hemispherical solid immersion lens is mounted on the bare surface of the sample to enhance the spatial resolution and the collection efficiency of single-dot emission in a low-temperature $(T=5 \mathrm{~K})$ scanning optical microscope. ${ }^{7}$ Despite the quite large QD density $\left(\approx 10^{10} \mathrm{~cm}^{-2}\right)$ and the large number of dots in the focal spot area, single QD transitions can be identified by their spectral signatures. A weak magnetic field of a few tens of milli-Tesla can be applied in Voigt or Faraday configuration using permanent magnets.

To investigate the mechanisms of spin injection, the QDs are excited with energy tunable picosecond $(\approx 2 \mathrm{ps})$ laser pulses from a frequency doubled optical parametric oscillator with a repetition time of $13 \mathrm{~ns}$. A delay line can be used to divide a single pulse into one co- and one cross-polarized pulses. Time-resolved experiments to observe slower dynamics (optical pumping of electron and nuclei) are performed using a modulated tunable continuous wave $(\mathrm{cw})$ dye laser. Laser pulses of controllable duration and polarization are created using Acousto-Optic Modulators or an Electro-Optic modulator with rise times of about $10 \mathrm{~ns}$. The collected PL is dispersed by a $1 \mathrm{~m}$ double monochromator before being detected by a CCD camera or a fast avalanche photodiode in conjunction with a time-correlated photon-counting unit with an overall time resolution of about $50 \mathrm{ps}$. In cw experiments the polarization rate of the PL is measured using a birefringent prism to separate the $\sigma+$ from the $\sigma-$ component and to detect them at the same time on different areas of the CCD camera.

\section{NUCLEAR SPIN POLARIZATION IN II-VI QUANTUM DOTS}

In a singly charged QD under the injection of spin polarized electrons, a nuclear spin polarization builds up by integration over many mutual spin flip-flops of the confined electrons and the lattice nuclei. This nuclear magnetic field modifies the coherent electron spin dynamics and consequently the average polarization of the PL of the $X^{-}$. The knowledge of the nuclear spin polarization can then be used to estimate the resident electron spin polarization. In this section we want to estimate the order of magnitude of the nuclear spin polarization that can build up in a II-VI QD and its influence on the spin dynamics of a confined electron.

The dominant contribution to the coupling between the confined electron and the nuclear spins originates from a Fermi contact hyperfine interaction. This interaction can be written as ${ }^{8}$

$$
H_{\mathrm{hf}}=v_{0} \sum_{i} A_{i}^{I}\left|\psi\left(R_{i}\right)\right|^{2}\left(I_{z}^{i} \sigma_{z}+\frac{I_{+}^{i} \sigma_{-}+I_{-}^{i} \sigma_{+}}{2}\right),
$$

where $R_{i}$ is the position of the nuclei $i$ with spin $I^{i}$ and hyperfine interaction constant $A_{i}^{I} . \sigma$ and $I^{i}$ are the spin operators of the electron and nuclei respectively. $v_{0}$ is the volume of the unitary cell containing $Z=2$ nuclei (one $\mathrm{Cd}$ and one Te). This Hamiltonian can be decomposed in a static part affecting the energy of the electron and nuclear spins and a dynamical part proportional to $\left(I_{+}^{i} \sigma_{-}+I_{-}^{i} \sigma_{+}\right)$, allowing for the transfer of angular momentum between the electron and nuclear spin system. The static part of the hyperfine interaction leads to the notion of effective magnetic field, either seen by the electron due to the spin polarized nuclei (Overhauser field $\vec{B}_{N}$ ), or by a nucleus at position $R_{i}$ due to a spin polarized electron (Knight field $\vec{B}_{e}^{i}$ ). These fields are defined by the electron-nuclei interaction energy:

$$
H_{\mathrm{hf}}=g_{e} \mu_{B} \vec{\sigma} \cdot \vec{B}_{N}=-\sum_{i} \mu_{I}^{i} \vec{I}^{i} \cdot \vec{B}_{e}^{i},
$$

where $g_{e}$ is the Lande factor of the electron and $\mu_{I}^{i}$ is the magneton of nucleus $i$ with spin $I^{i}$ defined by $\mu_{I}^{i}=\hbar \gamma_{I}^{i} I$, with $\gamma_{I}^{i}$ the gyromagnetic ratio of the nucleus $i$.

\section{A. Overhauser field in a CdTe/ZnTe quantum dot}

The maximum Overhauser field resulting from a complete polarization of the nuclei $B_{N}^{\max }$ is defined by intrinsic parameters characterizing the material and the hyperfine interaction inside the material. ${ }^{9,10}$ The Overhauser field can be written as

$$
B_{N}=\frac{v_{0}}{g_{e} \mu_{B}} \sum_{i}^{N_{I}} A_{i}^{I}\left|\psi\left(R_{i}\right)\right|^{2}\left\langle I_{z}^{i}\right\rangle
$$

where the sum runs over $N_{I}$, the number of nuclei carrying a spin $I$. When all the $\mathrm{Cd}$ and $\mathrm{Te}$ nuclear spins are polarized and if we assume a homogeneous electron wave function $\psi(R)=$ $\sqrt{2 /\left(v_{0} N_{L}\right)}$, with $N_{L}$ the total number of nuclei in the QD, the nuclear field reads

$$
B_{N}^{\max }=\frac{1}{g_{e} \mu_{B}}\left(I^{\mathrm{Cd}} A^{\mathrm{Cd}} p^{\mathrm{Cd}}+I^{\mathrm{Te}} A^{\mathrm{Te}} p^{\mathrm{Te}}\right) .
$$

The nuclear spin of $\mathrm{Cd}$ and $\mathrm{Te}$ are $I^{\mathrm{Cd}}=I^{\mathrm{Te}}=1 / 2$ and their corresponding abundance $p^{I}=N_{I} / N_{L}$ are $p^{\mathrm{Cd}}=0.25$ and $p^{\mathrm{Te}}=0.08$ (see Table I). Taking the hyperfine coupling constants $A^{\mathrm{Cd}} \approx-31 \mu \mathrm{eV}, A^{\mathrm{Te}} \approx-45 \mu \mathrm{eV}$ from Ref. 5 and an average electron Lande factor $g_{e} \approx-0.5,{ }^{11,12}$ we obtain $B_{N}^{\max } \approx 200 \mathrm{mT}$.

The Overhauser field really obtained in a QD under optical pumping $B_{N}$ is proportional to the average nuclear spin polarization $\left\langle I_{z}\right\rangle$ and reaches $B_{N}^{\max }$ when $\left\langle I_{z}\right\rangle=1 / 2$. As the electron Lande factor in CdTe/ZnTe QDs is negative and the

TABLE I. Isotopic abundance, nuclear spin $I$, and magneton of the nucleus $\mu_{I}$ for $\mathrm{Cd}$ and Te alloys. ${ }^{13} \mu_{I}$ is given in unit of the nuclear magneton $\mu_{N}$.

\begin{tabular}{lccc}
\hline \hline & Abundance (\%) & $I$ & $\mu_{I}$ \\
\hline${ }^{111} \mathrm{Cd}$ & 12.75 & $1 / 2$ & -0.5943 \\
${ }^{113} \mathrm{Cd}$ & 12.26 & $1 / 2$ & -0.6217 \\
${ }^{123} \mathrm{Te}$ & 0.87 & $1 / 2$ & -0.7357 \\
${ }^{125} \mathrm{Te}$ & 6.99 & $1 / 2$ & -0.8871 \\
\hline \hline
\end{tabular}


hyperfine constants are negative, the sign of $B_{N}$ is fixed by the sign of $\left\langle I_{z}\right\rangle$ which is given by the average electron spin polarization $^{9,14}\left\langle S_{z}\right\rangle$ along the QD growth axis $z\left(\left\langle S_{z}\right\rangle=1 / 2\right.$ for fully polarized spin up electrons). In the present study, the resident electron is pumped down $\left(\left\langle S_{z}\right\rangle \leqslant 0\right)$ for a $\sigma+$ excitation (spin $|\downarrow\rangle$ electron) in the presence of a positive external magnetic field. Thus, a $\sigma+$ excitation leads to an Overhauser field $B_{N}$ antiparallel to the applied magnetic field $\left(B_{N}<0\right)$.

\section{B. Knight field in a CdTe/ZnTe quantum dot}

At zero external magnetic field, the formation of a nuclear spin polarization is only possible if the effective field induced by the electron spin on the nuclei exceeds the local field $B_{l}$ created by the nuclear dipole-dipole interaction. ${ }^{14}$ The Knight field is inhomogeneous across the nuclear ensemble because the electron wave function is not constant across the QD. In the core of the QD where the Knight field is the strongest, the spin diffusion induced by the nuclear dipole-dipole coupling is suppressed and it is there that the nuclear spins may become polarized. The magnitude of the time averaged Knight field for a nucleus with a hyperfine constant $A^{I}$ at the position $R_{i}$ is given by

$$
B_{e}^{i}=-v_{0} \frac{A^{I}}{\mu_{I}}\left|\psi\left(R_{i}\right)\right|^{2}\left(\left\langle S_{z}\right\rangle f_{e}\right),
$$

where $f_{e}$ is the probability that the dot is occupied by an electron. Considering a constant electron-nuclei overlap [homogeneous wave function $\psi(R)=\sqrt{2 /\left(v_{0} N_{L}\right)}$ ] one can obtain $^{8,15}$ the maximum Knight field for nuclei with a hyperfine constant $A^{I}$ :

$$
B_{e}^{\max }=-\frac{A^{I}}{N_{L} \mu_{I}} .
$$

With $\mu_{\mathrm{Cd}} \approx-0.6$ and a total number of nuclei in the QD assumed to be $N_{L} \approx 8 \times 10^{3}, B_{e}^{\max } \approx 100 \mathrm{mT}$ is derived for $\mathrm{Cd}$ nuclei. A Knight field of $10 \mathrm{mT}$ is typical for InAs/GaAs QDs. ${ }^{16}$ The difference is a consequence of the smaller QD size in II-VI materials. However, $B_{e}$ follows the distribution of the electron wave function in the dot leading to a nuclear sitedependent field varying across the dot and in optical pumping experiments, only a weighted-averaged value of the Knight field can be accessible.

\section{Dynamic nuclear spin polarization}

Under circularly polarized $\mathrm{cw}$ excitation, the rate equation describing the nuclear spin polarization $I_{z}$ in a singly negatively charged QD can be written as ${ }^{17}$

$$
\frac{\partial I_{z}}{\partial t}=\frac{1}{T_{1 e}}\left[\frac{4}{3} I(I+1) S_{0}-I_{z}\right]-\frac{1}{T_{d d}} I_{z}-\frac{1}{T_{r}} I_{z} .
$$

The last term on the right side of the equation accounts for any spin relaxation mechanism except the dipole-dipole interaction between nuclei which is described by the relaxation time $T_{d d}$. The first term corresponds to the transfer of angular momentum between the spin of the electron and the nuclear spin bath, with $S_{0}$ the polarization rate of the injected electrons and $T_{1 e}$ the probability of an electron-nuclei spin "flip-flop" given by ${ }^{18}$

$$
\frac{1}{T_{1 e}}=\frac{1}{T_{1 e}^{0}} \frac{1}{1+\left(\Delta E_{e Z} / \hbar\right)^{2} \tau_{e}^{2}} .
$$

Here $\tau_{e}$ is the correlation time of the electron-nuclear spin interaction. ${ }^{17} \Delta E_{e Z}=g_{e} \mu_{B}\left(B_{\text {ext }}+B_{N}\right)$ is the electron Zeeman splitting which depends on the external magnetic field $B_{\text {ext }}$ and the effective nuclear field $B_{N}$. This term provides a feedback process mechanism between the spin transfer rate and the nuclear polarization leading to the enhancement of flip-flop processes when $B_{N}$ reduces the electron Zeeman splitting. This feedback is responsible for the bistability in the nuclear spin polarization observed under magnetic field in InAs/GaAs QDs. ${ }^{5,10,18} T_{1 e}^{0}$ is given by

$$
\frac{1}{T_{1 e}^{0}}=f_{e} \tau_{e}\left(\frac{E_{\mathrm{hf}}}{\hbar}\right)^{2}
$$

with

$$
E_{\mathrm{hf}}=\nu_{0} A_{i}^{I}\left|\psi\left(R_{i}\right)\right|^{2},
$$

the interaction energy between the electron and nuclear spin $I$ at position $R_{i} . T_{1 e}^{0}$ corresponds to the nuclear spin relaxation induced by the electron at zero electron splitting. For a homogeneous electron wave function $\left[\psi(R)=\sqrt{2 /\left(v_{0} N_{L}\right)}\right]$ we obtain $1 / T_{1 e}^{0}=f_{e} \tau_{e}\left[2 A_{i}^{I} /\left(N_{L} \hbar\right)\right]^{2}$.

The contribution of the dipole-dipole interaction to the relaxation process is given, in the presence of an external magnetic field $B_{\text {ext }}$, by

$$
\frac{1}{T_{d d}}=\frac{1}{T_{d d}^{0}} \frac{B_{l}^{2}}{\left(B_{\mathrm{ext}}+B_{e}\right)^{2}+B_{l}^{2}},
$$

where $B_{l}$ is the local field describing the nuclear spin-spin interaction and $T_{d d}^{0}$ the characteristic time of this interaction at zero field. ${ }^{19-21}$ This formula describes the acceleration of the nuclear spin relaxation when an applied magnetic field compensates the Knight field.

The magnetic field dependence of the nuclear spin polarization for a single family of nuclei with an average hyperfine coupling $A_{\mathrm{av}}=-40 \mu \mathrm{eV}$ and abundance $p_{\mathrm{av}}=16 \%$ obtained by a numerical determination of the steady state of the rate equation (7) is presented in Fig. 1. In this model, the electron wave function is described by a Gaussian function in the QD plane and a constant function in the $z$ direction:

$$
\psi(\rho, z)=\frac{1}{\sqrt{L_{z}}} \frac{1}{\xi \sqrt{\pi}} e^{-\frac{\rho^{2}}{2 \xi^{2}}},
$$

where $L_{z}$ is the thickness of the QD and $\xi$ is the lateral extension of the electron wave function. For the numerical calculation, the Gaussian wave function is approximated by a seven steps function (inset of Fig. 1) and the seven coupled differential equations corresponding to the seven families of nuclear spins (i.e., with different Knight fields) are solved simultaneously.

The acceleration of the dipole-dipole interaction between the nuclear spins when the external field compensate the Knight field is responsible for the decrease of the nuclear spin polarization observed at low negative magnetic field (between $B_{z} \approx-50 \mathrm{mT}$ and $B_{z} \approx-100 \mathrm{mT}$ ). The position of this minimum depends on the average electron spin polarization 


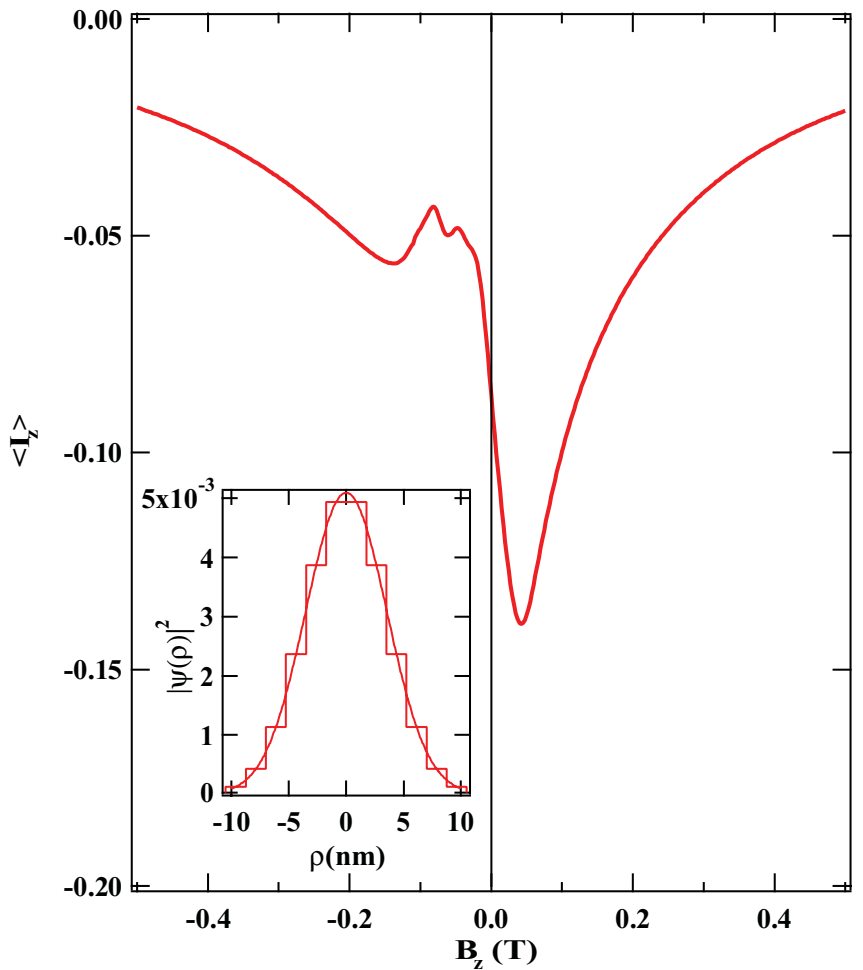

FIG. 1. (Color online) Nuclear spin polarization obtained from Eq. (7) for a single family of nuclei with an average hyperfine coupling $A_{\mathrm{av}}=-40 \mu \mathrm{eV}$ and abundance $p_{\mathrm{av}}=16 \%$ and with the parameters $f_{e}=0.4, S_{0}=-0.4, T_{d d}^{0}=5 \mu \mathrm{s}, B_{l}=2.5 \mathrm{mT}, d=2.5 \mathrm{~nm}, \xi=$ $5 \mathrm{~nm}, \tau_{e}=5 \mathrm{~ns}$, and $T_{r}=5 \mathrm{~ms}$. The inset shows the approximation of the Gaussian electron density with $\xi=5 \mathrm{~nm}$ and $L_{z}=2.5 \mathrm{~nm}$ by a step function used in the numerical calculation.

and is a measurement of the mean value of the Knight field. The nuclear spin polarization does not drop to zero because of the inhomogeneity of the Knight field: the condition $B_{\text {tot }}=0$ is satisfied only for a small number of nuclei at any given $B_{\text {ext }}$. Provided $B_{e} \gg B_{l}$, the majority of nuclei experience negligible change in depolarization.

The feedback process occurring when the Overhauser field compensates the applied magnetic field leads to a strong increase of the nuclear spin polarization at low positive magnetic field $\left(B_{z} \approx 50 \mathrm{mT}\right)$. This resonant effect is enhanced by a long electron correlation time $\tau_{e}$, that is, a weak broadening of the electron transition. This time of free coherent electron-nuclei precession is likely to be controlled in our experimental condition (i.e., chemically doped QDs under cw excitation) by the nonresonant optical injection of an electron-hole pair: we chose $\tau_{e}=5 \mathrm{~ns}$ in the calculation presented in Fig. 1.

The important variations of the nuclear spin polarization observed in Fig. 1 for a small varying magnetic field around $B_{z}=0 \mathrm{~T}$ are expected to significantly influence the spin dynamics of the resident electron. In particular, an increase of the relaxation rate of the electron spin should be observed when the external magnetic field compensates the Overhauser field increasing the influence of the fluctuating nuclear field.

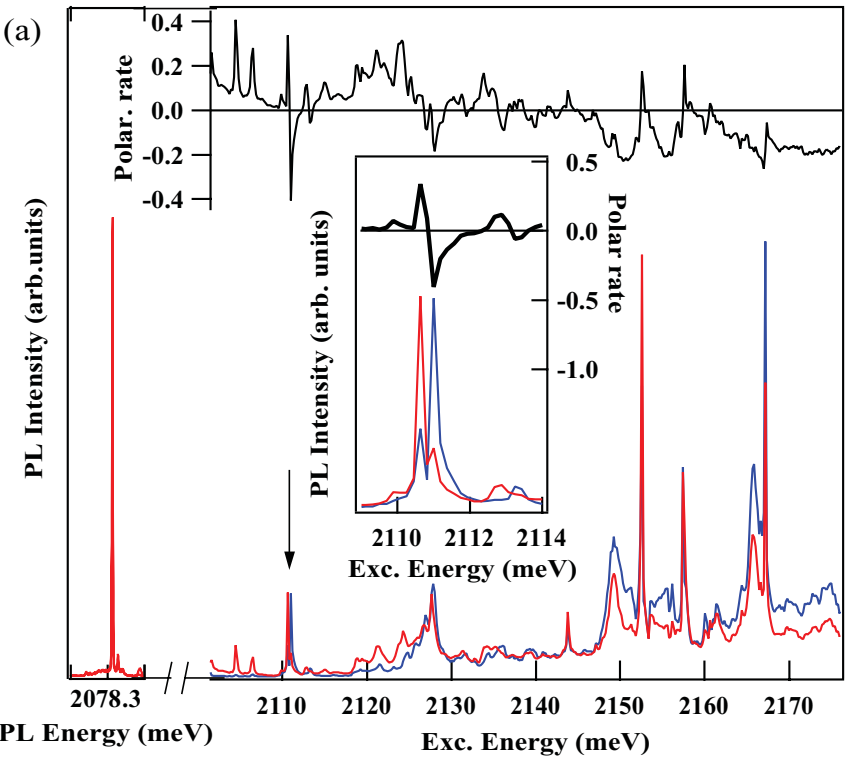

(b)

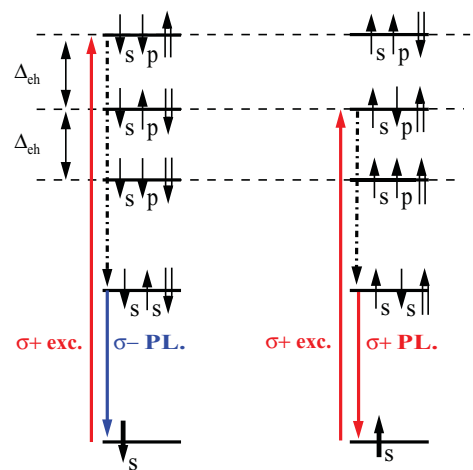

FIG. 2. (Color online) (a) PL and PLE spectra resolved in circular polarization under $\sigma+\mathrm{cw}$ excitation. The inset is a zoom on the polarized doublet in the PLE spectra, the polarization rate is also displayed. (b) Energy levels of the negative trion states. The electrons triplet state is split by the electron-hole exchange energy $\Delta_{\text {eh }}$. The electrons singlet state, also part of the $p$ shell is not represented here and the effect of the anisotropic exchange interaction on the energy levels is neglected. Left scheme: when exciting with $\sigma+$ light on the triplet state $\left|S=1, S_{z}=-1\right\rangle$, photon absorption occurs only if the resident electron is down. As demonstrated by Ware, ${ }^{22}$ during the excited trion relaxation, an electron-hole flip-flop process allowed by anisotropic exchange interactions results in a $\sigma-$ PL. Right scheme: when exciting with $\sigma+$ light on the triplet state $\left|S=1, S_{z}=0\right\rangle$, photon absorption occurs only if the resident electron is up. Fast relaxation from this state leads to $\sigma+\mathrm{PL}$.

\section{SPIN INJECTION IN NEGATIVELY CHARGED QUANTUM DOTS}

\section{A. Polarized fine structure of the excited state of the charged exciton}

In order to prepare the spin state of a resident carrier in a singly charged QD, spin polarized electron-hole pairs are injected through circularly polarized photoexcitation of an excited state of the QD. Low power PLE spectra on a singly negatively charged QD presented in Fig. 2 reveals intense absorption resonances for $X^{-}$with a strong polarization 
dependence. In general we find three distinctive features in these excitation spectra.

The first is a set of lower energy resonances that are strongly copolarized with the excitation laser. These transitions can be assigned to nominally forbidden transitions involving states with two $s$-shell electrons and an excited or delocalized hole. These transitions are particularly well observed in CdTe/ZnTe structures because of the weak valence band offset.

The second feature is a higher energy resonance that displays a fine structure doublet well resolved in circular polarization. As presented in Fig. 2(a), the PLE exhibits a strongly co- and then cross-polarized resonance as the laser energy increases around $2110 \mathrm{meV}$. As proposed by Ware et al. ${ }^{22}$ we can assign this doublet to the direct excitation of the two bright triplet states [see Fig. 2(b)] of the excited negatively charged exciton $\left(X^{-*}\right) . X^{-*}$ consists of an electron-hole pair in the $S$ shell and an electron in the $P$ shell. This doublet is a characteristic signature of the presence of a single electron in the QD ground state. We have found a triplet splitting $\Delta_{\mathrm{eh}}$ around $400 \mu \mathrm{eV}$ changing from dot to dot. This is higher than the values found in InAs QDs, in agreement with the stronger exchange interaction in our II-VI QD system.

For an excitation above the $X^{-*}$ triplet states, a series of excited states and an absorption background with a significant negative circular polarization rate are observed. ${ }^{23}$ As we will discuss in the next section, this condition of excitation can be used to perform an optical pumping of the resident electron spin.

\section{B. Kinetics of the degree of circular polarization}

As a probe of the resident electron spin orientation, we will use the amplitude of the negative circular polarization of the charged QDs. ${ }^{24-26}$ In the case of $X^{-}$, the circular polarization of the emitted light reflects both the spin of the resident electron before the absorption of a photon and the spin of the hole before emission. Negative polarization of $X^{-}$implies that the hole spin has flipped prior to recombination and that a spin flipped hole contributes to the $X^{-}$formation with a higher probability than a nonflipped hole. This process also leads to an optical pumping of the resident electron spin.

The kinetics of the degree of circular polarization observed in time-resolved PL experiments reflects the mechanisms of spin injection. The time dependence of the polarisation rate $\rho_{c}$ under quasiresonant pulsed excitation $(\approx 2 \mathrm{ps})$ is displayed in Fig. 3. As we discussed for the PLE spectra of the QD presented in Fig. 2, the lowest excited state ( $E_{1}$ in Fig. 3) is fully copolarized. It corresponds to the negatively charged state with two electrons in the $S$ shell and a hole in a higher shell. It is spin selective as the spin of the two electrons in the $S$ shell have to be opposite: under $\sigma+$ excitation, an absorption only occurs if the resident electron is $|\uparrow\rangle$. The fast initial decay (in the range of $100 \mathrm{ps}$ ) of the polarization under excitation on $E_{1}$ is attributed to a relaxation of the hole spin on the high energy shell while the slower decay (in the range of $5 \mathrm{~ns}$ ) is attributed to relaxation of the hole spin when it is in the $S$ shell. This fast hole spin relaxation is similar to the one observed in $\mathrm{CdSe} / \mathrm{ZnSe}$ QDs. ${ }^{27}$

Resonant $\sigma+$ excitation on the charged exciton triplet states ( $E_{2}$ in Fig. 3) results in the creation of an excited trion $\Uparrow_{P} \downarrow P \uparrow s$
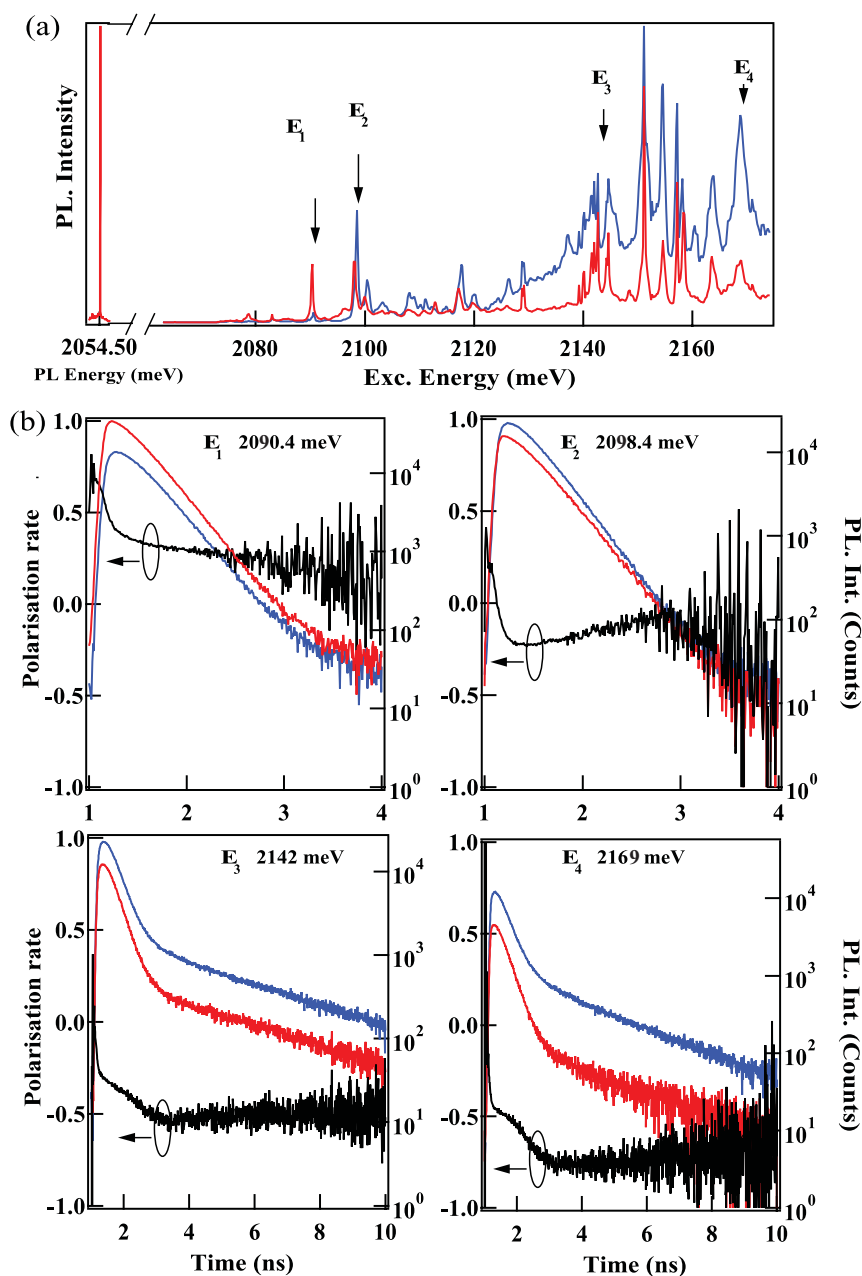

FIG. 3. (Color online) (a) PL and PLE spectra of a singly charged quantum dot. (b) Time evolution of the co-polarized PL (red line), the cross-polarized (blue line) PL, and the degree of circular polarization (black line) under unmodulated circularly polarized pulsed excitation for four different excitation energies $\left(E_{1}\right.$ to $\left.E_{4}\right)$. A clear negative polarization rate is observed when the excitation energy is higher than the triplet states of the excited charged exciton $X^{-*}\left(E_{2}\right)$.

or $\Uparrow_{P} \downarrow_{P} \downarrow_{S}$ depending on the spin of the resident electron [see Fig. 2(b)]. Under excitation on $E_{2}$ we observe a fast decay of the initial positive polarization rate: the polarization rate becomes negative in a few tens of picoseconds. This evolution reflects the spin dynamics of $X^{-*}$. The excited state $\Uparrow_{P} \downarrow_{P} \uparrow s$ can relax quickly to the ground trion state, while for the state $\Uparrow_{P} \downarrow_{P} \downarrow_{S}$ relaxation to the ground state is forbidden until an electron-hole flip-flop occurs through anisotropic exchange interaction. ${ }^{28}$ Therefore, relaxation from $X^{-*}$ results in a positive polarization rate at short delays and negative at longer delays.

For higher excitation energies ( $E_{3}$ and $E_{4}$ in Fig. 4) a major part of the decay of the polarization rate takes place within the first 200 ps. The polarization rate becomes quickly negative and approaches a value of about $-30 \%$. It further decreases at longer time delay $(\approx 1 \mathrm{~ns})$ and reaches a steady state value lower than $-50 \%$. To understand the three regimes in the dynamics of the polarization rate, we have to consider $0 \mathrm{D}-2 \mathrm{D}$ 

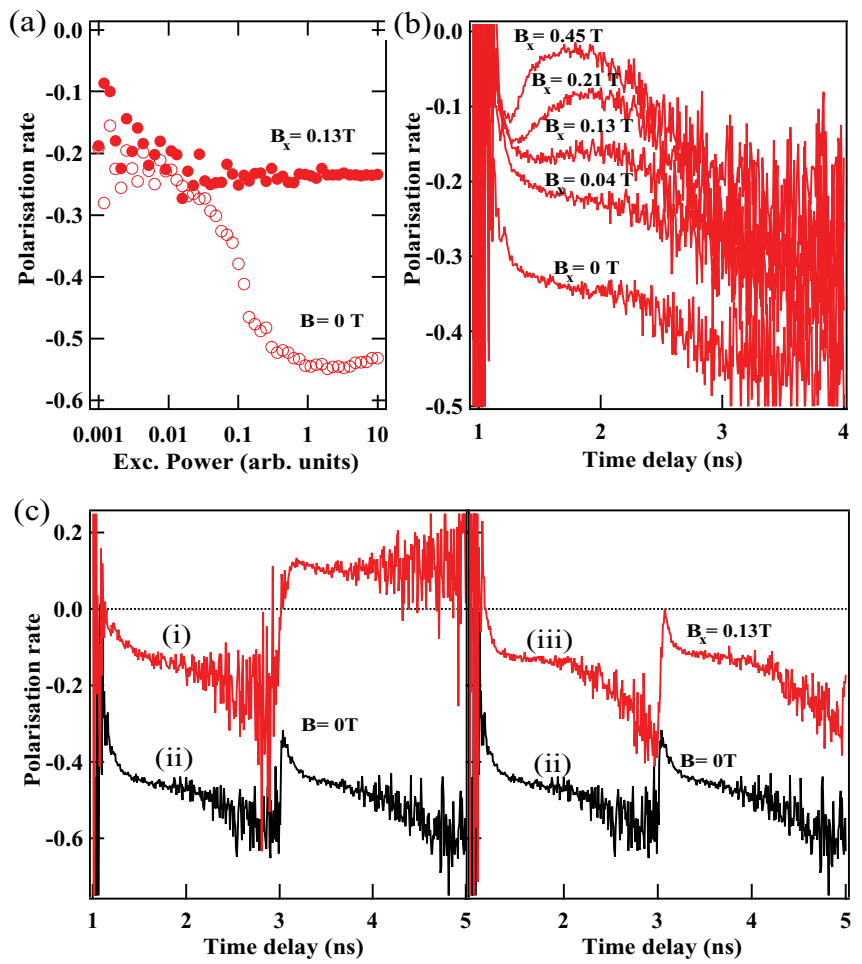

FIG. 4. (Color online) (a) Evolution of the degree of circular polarisation as a function of $\mathrm{cw}$ excitation power. Empty circles are zero field measurements, filled circles are measurements in a transverse magnetic field $B_{x}=0.13 \mathrm{~T}$. (b) Time evolution of the degree of circular polarization under pulsed ( 2 ps) quasiresonant excitation for different transverse magnetic fields. (c) Time evolution of the degree of circular polarization with a two-pulse-excitation sequence: the pulses are separated by 2 ns. (i) is obtained with cross-polarized pulses at zero field. (ii) is obtained with copolarized pulses at zero field. (iii) is obtained with copolarized pulses and a transverse magnetic field $B_{x}=0.13 \mathrm{~T}$.

cross transitions where the electron is injected in the dot and the hole in the wetting layer. Such transitions are particularly important in our system presenting a weak valence band offset. The spin of the hole is randomize before its capture by the QD, whereas the electron spin is conserved (spin $|\downarrow\rangle$ for $\sigma+$ excitation). The captured bright excitons (i.e., without hole spin flip) or dark excitons (i.e., after a hole spin flip) relax to form the hot trion $X^{-*}$. For an unpolarized resident electron, four possible channels are then possible for the relaxation of the hot trion formed by a $\sigma+$ excitation:

(1) $\uparrow_{s \downarrow} \uparrow \uparrow_{p} \uparrow_{s} \downarrow_{s} \uparrow \longmapsto \sigma+$ and $\uparrow_{s}$,

(2) $\uparrow_{s} \downarrow_{p} \Downarrow \longmapsto \uparrow_{s} \downarrow_{s} \Downarrow \longmapsto \sigma-$ and $\downarrow_{s}$,

(3) $\downarrow_{s} \downarrow_{p} \uparrow \stackrel{\delta_{a}}{\longmapsto} \downarrow_{s} \uparrow{ }_{s} \Downarrow \longmapsto \sigma-$ and $\downarrow_{s}$,

(4) $\downarrow_{s} \downarrow_{p} \Downarrow \stackrel{\tau_{h}}{\longmapsto} \downarrow_{s} \downarrow_{p} \Uparrow \stackrel{\delta_{a}}{\longmapsto} \uparrow_{s} \downarrow_{s} \Downarrow \longmapsto \sigma-$ and $\downarrow_{s}$,

where $\delta_{a}$ is an anisotropic electron-hole exchange interaction term responsible for the flip-flop of the electron-hole pair in the QD excited state and $\tau_{h}$ the spin flip time of a hole in the $S$ shell. The realization of (1) and (2) is proportional to the probability for the resident electron to be $|\uparrow\rangle$ and does not require any spin flip of the hot trion: they take place in the picosecond range. (3) and (4) depend on the probability of having the resident electron $|\downarrow\rangle$ and involve spin flips of the hot trion. These last two channels [(3) and (4)] lead to the appearance of a negative circular polarization rate with two time scales, one in the tens of picoseconds range governed by $\delta_{a}$ and one in the nanoseconds range governed by $\tau_{h}$.

If the spin relaxation rate of the resident electron is longer than the optical excitation rate, cumulative effects lead to the optical orientation of the electron spin. At this stage we have to notice that the observation of negative circular polarization does not necessarily mean that the resident electron is polarized but a variation of its polarization will cause a change in the negative circular polarization rate.

\section{ELECTRON SPIN OPTICAL ORIENTATION}

\section{A. Orientation of the spin of the electron}

The presence of optical pumping of the resident electron is confirmed by the power dependence of the negative circular polarization rate obtained under $\mathrm{cw}$ excitation [Fig. 4(a)]. As the pump power intensity is increased, we observe a rapid growth of the negative circular polarization with a saturation at about $-55 \%$. This reveals the progressive orientation of the resident electron spin by the exciting beam. The remaining polarization rate observed at low excitation power or in a weak transverse magnetic field is attributed to the different processes of carrier relaxation discussed in Sec. IV. In the optical pumping regime (i.e., without transverse magnetic field and at large excitation intensity), the measurement of the negative circular polarization gives an estimate of the degree of the electron spin polarization.

The electron spin memory can be significantly erased by a weak magnetic field [see Fig. 4(b)] applied in the plane of the QD. At $B_{x} \approx 0.1 \mathrm{~T}$, all the contribution of the electron spin polarization to the negative circular polarization rate has disappeared. Despite the weak transverse component of the hole $g$ factor, a further increase of the transverse magnetic field can induce a precession of the confined hole spin during the lifetime of the negatively charged exciton. At high field, this precession depolarizes the hole spin and finishes to destroy the average negative circular polarization of the $X^{-}$. This effect is observed in Fig. 4(b) as an oscillation of the polarization rate for a transverse field larger than $0.1 \mathrm{~T}$. This oscillation corresponds to the first period of precession of a spin polarized hole injected at $t=0 \mathrm{~ns}$. The decrease of the polarization rate at long time delay corresponds to the late recombination of spin flipped holes stored as dark excitons in the triplet state of $X^{-*}$.

The dynamics of the pumping and relaxation of the resident electron spin can be estimated through the time evolution of the polarization rate when the QD is excited by a sequence of two circularly polarized picosecond laser pulses. The results of the experiments using equal intensities for the two pulses are shown in Fig. 4(c). When the QD is excited with circularly copolarized pulses (ii), a large average negative circular polarization is observed for both PL pulses. However, in the case of excitation by cross-polarized beams (i), the average PL polarization vanishes. These results directly demonstrate that the spin orientation created by the first pulse affects the polarization of the PL excited by the second one. It means that after recombination of the electron-hole pair, the 
information about the polarization of the excitation is stored in the orientation of the resident electron spin. In addition, we notice that the polarization rate is identical for the two pulses in the excitation sequence. As these pulses are separated in time by either 2 or $11 \mathrm{~ns}$ this suggest that the relaxation time of the electron exceeds by far the laser pulses repetition rate $(\approx 13 \mathrm{~ns})$. Consequently, the resident electron can be fully depolarized by a weak transverse magnetic field. A significant decrease of the negative polarization rate is observed for both pulses in a transverse field $B_{x}=0.13 \mathrm{~T}$ (iii) confirming the influence of the optical pumping of the electron spin on the negative polarization rate.

\section{B. Dynamics of the electron spin orientation}

In the presence of optical pumping, the degree of negative circular polarization of $X^{-}$reflects the spin polarization of the resident electron. The dynamics of its optical orientation can then be revealed by the observation of the negative polarization rate under modulated circularly polarized excitation. As presented in Fig. 5(a), the negative polarization (a)

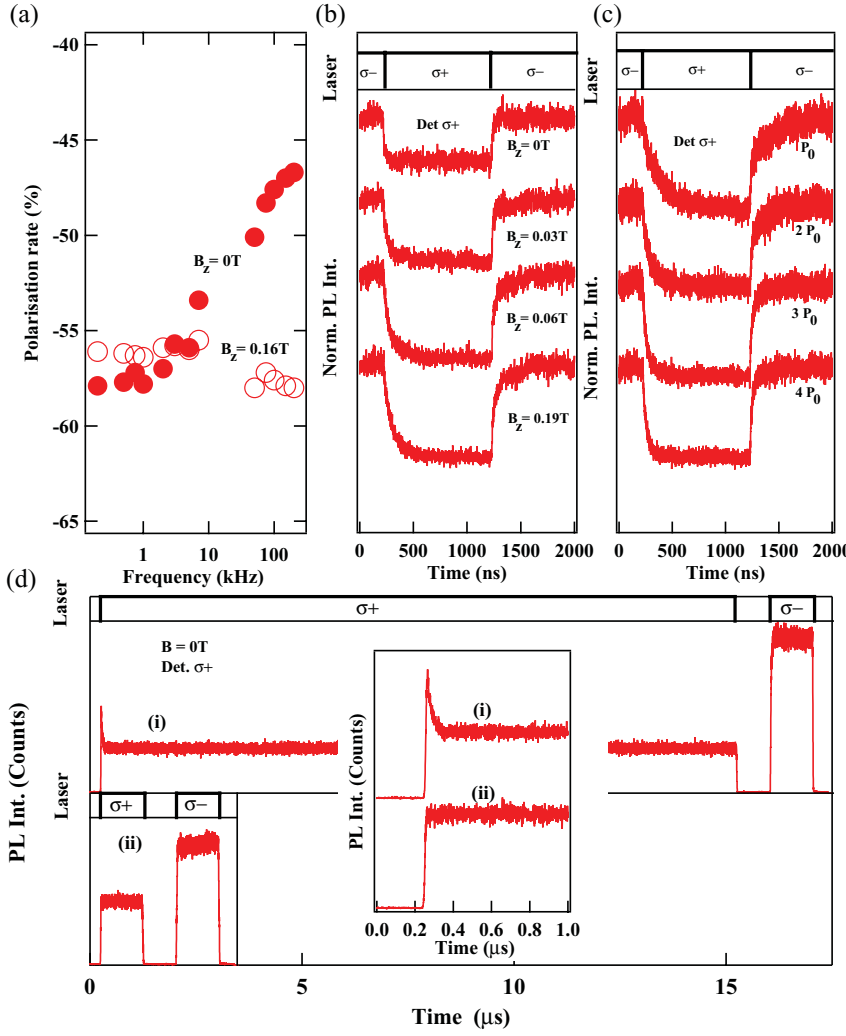

FIG. 5. (Color online) (a) Evolution of the NCP with the frequency of the $\sigma+/ \sigma-$ modulation of the excitation laser tuned on an excited state above the charged exciton triplet states at zero magnetic field and at a field $B_{z}=0.16 \mathrm{~T}$ applied along the QD growth axis $z$. Time evolution of the $\sigma+$ PL excited alternatively with $\sigma+$ or $\sigma-$ light for different magnetic fields applied in Faraday geometry (b) and for different excitation intensities and a fixed magnetic field $B_{z}=0.19 \mathrm{~T}$ (c). (d) Time evolution of the $\sigma+$ PL excited alternatively with $\sigma+$ and $\sigma-$ light trains. The excitation sequence are displayed above the spectrum. The inset is a zoom on the fast transient at short time delay. strongly depends on the modulation frequency: an increase of the degree of circular polarization when the modulation frequency is decreased is observed at $B=0 \mathrm{~T}$. This modulation frequency dependence is canceled by a magnetic field of $B_{z}=0.16 \mathrm{~T}$ applied along the QD growth axis. As already observed in InAs QDs, this behavior is a fingerprint of the coupling of the electron to a fluctuating nuclear field. ${ }^{29}$

The QD contains a finite number $N_{I}$ of nuclei carrying a spin, which means that statistically the number of spins parallel and antiparallel in any given direction differs by a value $\sqrt{N_{I} / 3}$. The result is an effective magnetic field $B_{f}$, oriented in a random direction. This field will induce a precession of the spin of the electron for every $B_{f}$ not aligned along the QD growth axis $z$. $B_{f}$ can be estimated for a CdTe QD. Assuming a homogeneous envelope function for the electron $\psi(R)=$ $\sqrt{2 /\left(v_{0} N_{L}\right)}, B_{f}$ is given by ${ }^{19}$

$$
\begin{aligned}
B_{f}^{2}= & \frac{2}{\left(g_{e} \mu_{B} \sqrt{N_{L}}\right)^{2}}\left[I_{\mathrm{Cd}}\left(I_{\mathrm{Cd}}+1\right) A_{\mathrm{Cd}}^{2} p_{\mathrm{Cd}}\right. \\
& \left.+I_{\mathrm{Te}}\left(I_{\mathrm{Te}}+1\right) A_{\mathrm{Te}}^{2} p_{\mathrm{Te}}\right] .
\end{aligned}
$$

For our estimation of $N_{L}=8000$ one obtain $B_{f} \approx 12 \mathrm{mT}$. The electron spin precession frequency in the frozen nuclear spin fluctuation $B_{f} \approx 12 \mathrm{mT}$ is $\approx 80 \mathrm{MHz}$. This frequency can be smaller than the rate of optical injection of the spin polarized carriers at high excitation intensity, allowing an optical pumping of the electron spin even in the presence of nuclear spin fluctuations.

At high modulation frequency of the polarization and low excitation intensity, a dynamic nuclear spin polarization does not have time to build up. Over time scales less than $1 \mu \mathrm{s}$, the electron is exposed to a snapshot of $B_{f}$ where the nuclear spin configuration remains frozen. In the absence of an external magnetic field, only this internal field $B=B_{f}$ acts on the electron. For a randomly oriented nuclear spin system, the electron spin polarization quickly decays to $1 / 3$ of its initial value due to the frozen nuclear field. ${ }^{30}$ This decay is not a real relaxation process as the electron coherently evolves in a frozen nuclear spin configuration. On an averaged measurement, a fast decay of the electron polarization on a characteristic time scale $t \approx h /\left(g_{e} \mu_{B} B_{f}\right)$ is expected. ${ }^{30}$ In the absence of nuclear spin polarization, the influence of the fluctuating nuclear field can be suppressed by applying an external magnetic field. For sufficiently large external fields, the nuclear spin fluctuations does not contribute significantly to the total field, $B_{\text {tot }}=B_{\text {ext }}+B_{f}$, and the electron-spin polarization is preserved.

At small modulation frequencies of the polarization or under cw excitation, nuclei can be dynamically oriented through flip-flop with the spin polarized resident electron. This nuclei orientation leads to the formation of an Overhauser field $B_{N}$ along the $z$ axis, which may be much larger than the in-plane component of the fluctuating field $\left(B_{f}\right)$. The electron now precesses around a nuclear field whose $z$ component dominates: the result is an increase of the average electron spin polarization compared to the case of a totally randomly oriented nuclear spin system. The effect of the Overhauser field is similar to an applied magnetic field along the $z$ axis 
allowing an optical orientation of the electron spin even at low excitation power.

This influence of the nuclear spin fluctuations in the optical pumping of the electron is confirmed by the magnetic field dependence of the time-resolved polarization rate obtained at high modulation frequency [Fig. 5(b)]. The increase of the polarization rate and the appearance of a transient with an applied external magnetic field along $z$ reflects an increase of the optical pumping efficiency of the electron. ${ }^{3}$ This optical pumping, which takes place in a few tens of nanoseconds, is promoted by the presence of the external field which can dominate the fluctuations of the Overhauser field. ${ }^{31}$ This short time scale component in the dynamics of the polarization of the spin of the electron becomes faster with the increase of the optical generation rate of spin polarized carriers and reach the nanosecond range [Fig. 5(c)].

Similarly to the application of an external magnetic field, the build up of a DNSP favors the electron spin polarization. This is confirmed by the following experiment: in Fig. 5(d) the $\sigma+$ PL has been time resolved using the two different excitation sequences displayed on each spectrum. In the sequence (ii), the excitation pulses are of equal length and power, and are short enough to prevent the creation of DNSP. In sequence (i), the difference of pulses length allows the creation of DNSP. The measurements show two striking differences. First, the average circular polarization, given by the difference of the PL intensity obtained under $\sigma-$ and $\sigma+$ excitation, is higher in (i) than in (ii). Second, the PL of (i) exhibits a fast PL transient at short delay reflecting an optical pumping of the electron spin [detail of this transient is shown in the inset of Fig. 5(d)]. These two features demonstrate that the Overhauser field created in (i) is strong enough to block the longitudinal decay of the electron spin by the fluctuating nuclear field.

\section{NUCLEAR SPIN POLARIZATION}

\section{A. Builtup of the nuclear spin polarization}

Direct evidence of the build up of a DNSP can be observed using sequences of pulses of long duration (tens of microseconda). As displayed in Fig. 6(a), the $\sigma+$ PL recorded under $\sigma+$ excitation presents first a fast transient with a drop of the intensity due to the orientation of the resident electron spin [a zoom of the transient at short delay is presented in Fig. 6(b)]. Then, a slower transient is observed: the $\sigma+\mathrm{PL}$ increases during a few microseconds reflecting a decrease of the absolute value of the negative polarisation (i.e., of the spin polarization of the resident electron) before it decreases again. This evolution has been predicted by Petrov et al..$^{32}$ and results from a destruction of the Overhauser field created at the end of the $\sigma$ - excitation pulse, and a build up of an Overhauser field in the opposite direction under $\sigma+$ excitation. During this process, the amplitude of the Overhauser field becomes zero and the electron spin is strongly affected by the nuclear spin fluctuations $B_{f}$.

As presented in Fig. 6(d), the speed of the destruction and build up of the nuclear polarization strongly increases with the increase of the excitation power. Simultaneously, the average negative polarization of the $X^{-}$, given by the
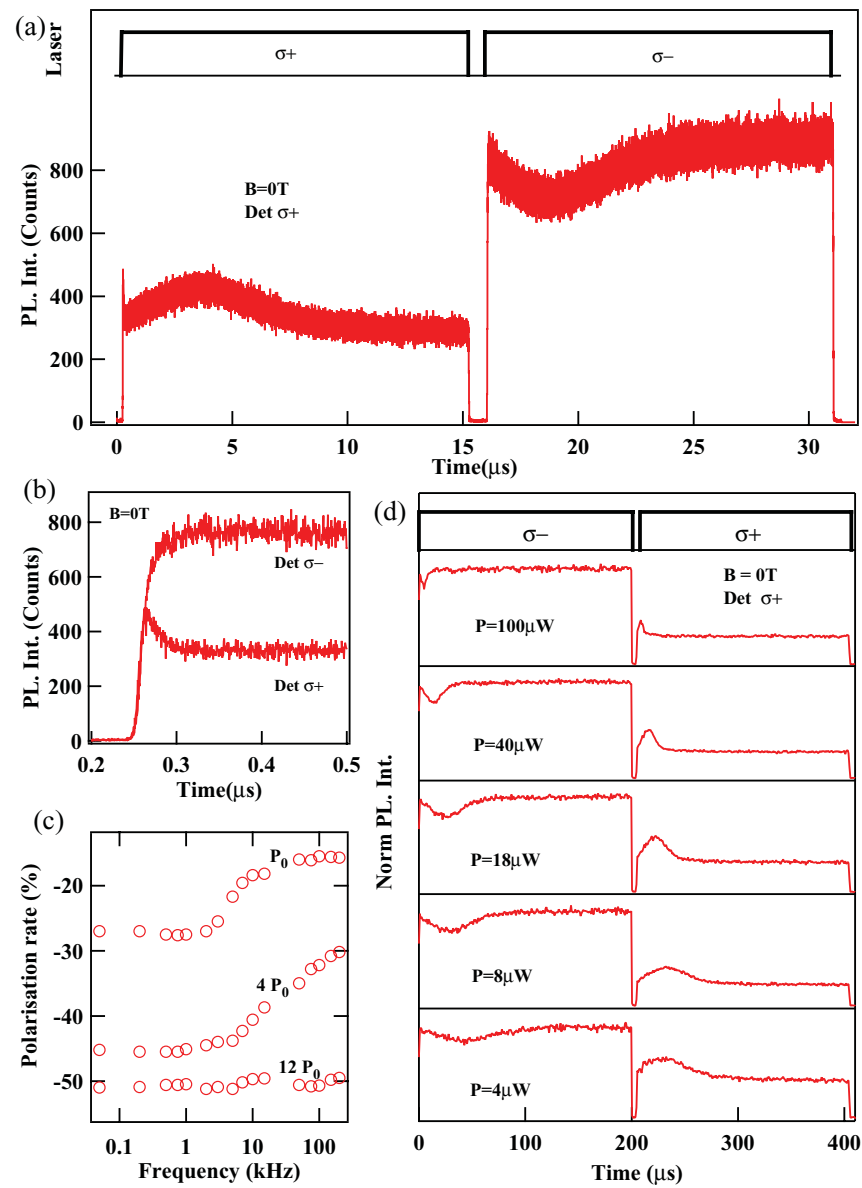

FIG. 6. (Color online) (a) Time evolution of the $\sigma+$ PL excited alternatively with $\sigma+/ \sigma-$ light trains. The excitation sequence is displayed above the spectrum. (b) Zoom on the transient corresponding to the optical pumping of the electron. The time evolution of the $\sigma-\mathrm{PL}$ recorded in the same conditions of excitation is also displayed. (c) Dependence of the the degree of circular polarisation of the QD PL on the frequency of the $\sigma+/ \sigma-$ modulation of the light for different excitation power. (d) Time evolution of the $\sigma+$ PL excited alternatively with $\sigma+/ \sigma-$ light for different excitation power.

intensity difference of the PL obtained under $\sigma+$ and $\sigma-$ excitation, increases. This effect is also directly observed in the modulation frequency dependence of the polarization rate displayed in Fig. 6(c). The modulation frequency required to suppress the nuclear spin polarization (i.e., to decrease the absolute value of the polarization) increases with the excitation intensity. At low excitation intensity, a formation time of the nuclear spin polarization of about $50 \mu$ s can be estimated from the modulation frequency dependence of the negative circular polarization. ${ }^{29}$ This is faster than in InAs/GaAs QDs where a pumping time ranging from a few hundred microseconds to $10 \mathrm{~ms}$ have been reported depending on the experimental conditions. ${ }^{10,33}$ At hight excitation power, the pumping rate of the nuclei becomes faster than the polarization modulation frequency and a stable negative polarization of about $-50 \%$ is obtained [Fig. 6(c)]. An increase of the value of the negative polarization with the excitation intensity is also observed, suggesting an increase of the average nuclear 

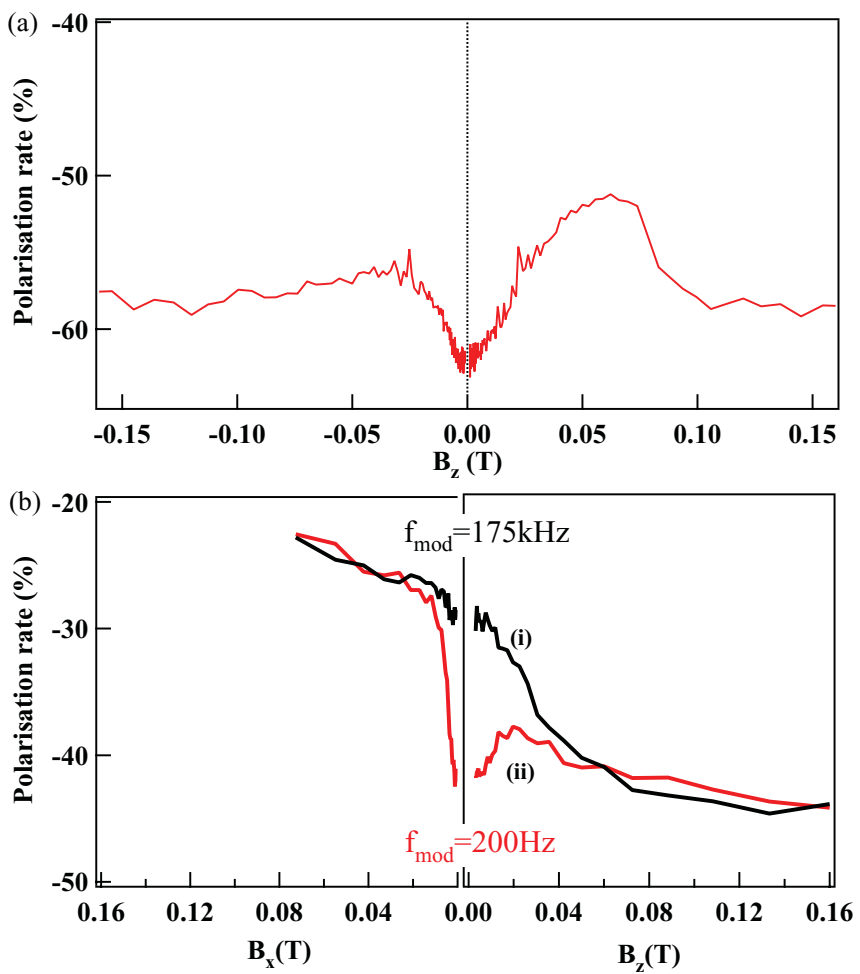

FIG. 7. (Color online) (a) Magnetic field dependence in Faraday configuration of the negative polarization rate under $\sigma+\mathrm{cw}$ excitation. (b) Magnetic field dependence of the negative polarization rate, on a different QD, in Voight (left) and Faraday (right) configuration under $\sigma+/ \sigma-$ modulated excitation at low $\left(f_{\bmod }=200 \mathrm{~Hz}\right)$ and high $\left(f_{\text {mod }}=175 \mathrm{kHz}\right)$ modulation frequency.

spin polarization and Overhauser field with the excitation intensity.

\section{B. Magnetic field dependence of the nuclear spin polarization}

A typical magnetic field dependence of the polarization rate of a singly charged QD under cw circularly polarized excitation, in the optical pumping regime, is presented in Fig. 7. Figure 7 (a) focuses on the Faraday geometry while the Voigt geometry and the influence of the modulation frequency of the polarization of the excitation beam are presented in Fig. 7(b). The asymmetry of the response in the Faraday geometry is a fingerprint of the presence of a nuclear spin polarization induced by the helicity of the excitation beam.

A striking feature of this magnetic field dependence is the small increase of the absolute value of the negative polarization around $B=0 \mathrm{~T}$. This is in opposition to what is usually observed in III-V semiconductor QDs, where a decrease of the electron spin polarization occurs at weak magnetic field because of the dominant contribution of the fluctuating nuclear field $B_{f}$. As presented in curve (i) of Fig. 7(b), a standard increase of polarization with magnetic field is restored in the absence of nuclear polarization (i.e., under polarization modulated excitation). This shows that the increase of the electron polarization around $B=0 \mathrm{~T}$ observed under $\mathrm{cw}$ excitation is linked to the DNSP: the nuclear spin fluctuations are strongly suppressed by the build up of a large Overhauser field.
The experiment presented in Fig. 7(a) was carried out under cw $\sigma+$ excitation, pumping the resident electron down. This leads to an average polarization of nuclei with $\left\langle I_{z}\right\rangle<0$ and an Overhauser field $B_{N} \leqslant 0$. For $B_{z} \geqslant 0$ we observe around $50 \mathrm{mT}$ an increase of the circular polarization rate of $10 \%$ which reflects a depolarization of the resident electron. This behavior is attributed to the compensation of the Overhauser field by the external Faraday field $\left(B_{z}=-B_{N}\right)$. As the electron precesses around the total field $B_{\text {tot }}=B_{z}+B_{N}+B_{f}$, the electron dynamics is then governed by the nuclear spin fluctuations $B_{f}$, resulting in a depolarization of the resident electron.

Considering the left panel of Fig. 7(a) (corresponding to $\left.B_{z} \leqslant 0\right)$, we observe at $B=0 \mathrm{~T}$ a maximum in the electron polarization, then a decrease of about $5 \%$ in the first $25 \mathrm{mT}$ followed by a small increase at larger fields. This extremum around $B_{z}=-25 \mathrm{mT}$ also reflects a depolarization of the resident electron spin. This depolarization is attributed to a compensation of the Knight field by the external magnetic field. The nuclear field is then close to zero and the electron dynamics is ruled by the sum of $B_{z} \approx 25 \mathrm{mT}$ and the nuclear spin fluctuations $B_{f}$. The effect of $B_{f}$ is not negligible at $25 \mathrm{mT}$. As observed in the experiment of Fig. 5(b), in the absence of DNSP, the polarization of the PL continuously increases as the Faraday magnetic field is increased from 0 to $60 \mathrm{mT}$.

This influence of the Knight and Overhauser fields is further confirmed by the following experiment: We present on the right panel of Fig. 7(b) a measurement where we have studied the polarization rate as a function of the magnetic field $B_{z}$ under modulated excitation. The light is modulated $\sigma+/ \sigma-$ at two different rates: (i) $175 \mathrm{kHz}(\approx 3 \mu \mathrm{s}$ of $\sigma+$ exc., then $\approx 3 \mu$ s of $\sigma-$ exc. of equal intensity) and (ii) $200 \mathrm{~Hz}(\approx 2500 \mu$ s for a given polarization). Hence, at low power of excitation, DNSP is achieved in (ii) and not in (i). The detection is done on an APD synchronized with the modulation, and for a fixed circularly polarized detection. We measure a polarization rate by varying the excitation polarization and not the detection. Hence, this polarization rate is an average of the polarization rates measured in Fig. 7(a) for $B_{z}$ and $-B_{z}$. The magnetic field dependence for (ii) is consistent with the one observed in the cw regime, with an evolution ruled by the competition between the $B_{z}, B_{N}, B_{e}$, and $B_{f}$. On the other hand, the magnetic field dependence (i) is only controlled by the competition between $B_{z}$ and $B_{f}$. For sufficiently large external fields, the nuclear spin fluctuations $B_{f}$ do not contribute to the total field and the electron-spin polarization does not decay. The width at half maximum is $25 \pm 5 \mathrm{mT}$. This gives an order of magnitude of the fluctuating Overhauser field.

The magnetic field dependence of the polarization rate under $\mathrm{cw}$ excitation has been performed for $B_{z}>0$ for different excitation powers [Fig. 8(a)]. With increasing power, the minimum in the electron polarization is shifted to higher magnetic field, evidencing an increase of the polarization of the nuclei and of the resulting Overhauser field. At high excitation intensity a significant portion of the nuclei are polarized and the minimum of electron polarization is observed around $B_{z}=-B_{N}=100 \mathrm{mT}$ which would correspond to $50 \%$ of the maximum Overhauser field. However, the parameters of II-VI QDs used to estimate this maximum field are 

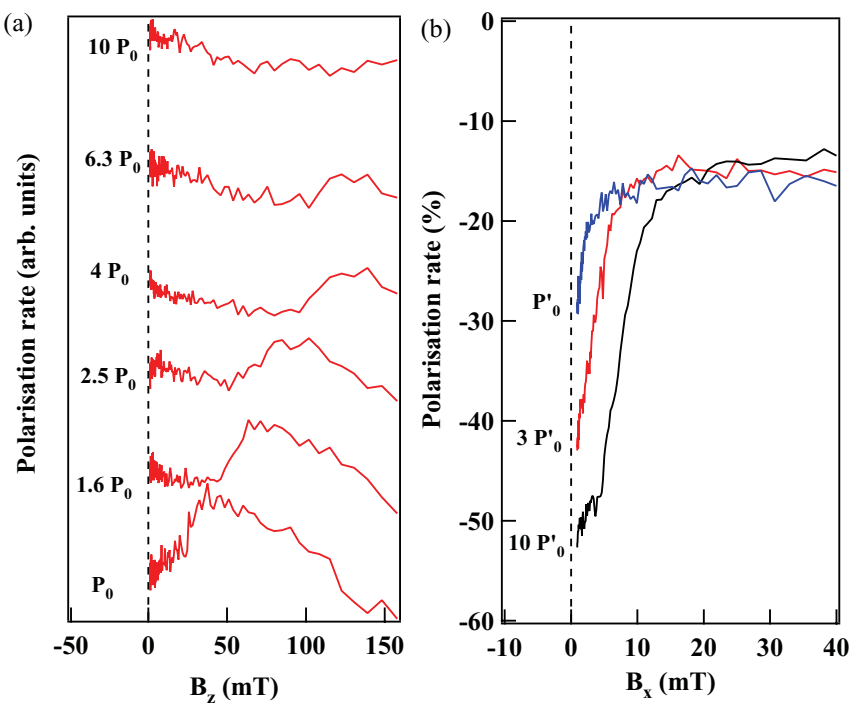

FIG. 8. (Color online) Excitation power dependence of the negative polarization rate under magnetic field. (a) Faraday configuration under $\sigma+\mathrm{cw}$ excitation. (b) Voigt configuration.

not known with precision and this percentage is subject to caution.

\section{DYNAMICS OF COUPLED ELECTRON AND NUCLEAR SPINS}

\section{A. Electron-nuclear spin system in a transverse magnetic field}

A transverse magnetic field dependence of the polarization rate of $X^{-}$is presented in the left panel of Fig. 7(b). As the transverse magnetic field is increased, we observe in the absence of DNSP (black curve, corresponding to fast $\sigma+/ \sigma-$ modulation), a progressive decrease of the negative polarization rate over the first $80 \mathrm{mT}$. For a spin polarized electron and in the absence of nuclear spin polarization, two processes can contribute to the observed Hanle depolarization of $X^{-}$. The first is a depolarization of the resident electron governed by the transverse relaxation time of the spin of the electron $T_{2}$ in an unpolarized nuclear spin bath (standard Hanle depolarization). This $T_{2}$ should give rise to a half width of the Hanle curve $B_{1 / 2}=B_{f} \approx 25 \mathrm{mT}$, deduced from the Faraday measurement in Fig. 7(b). The second mechanism is a precession of the hole during the charged exciton lifetime. This process is expected to play a role above $50 \mathrm{mT}$ as we have seen in the time-resolved polarization rates presented in Fig. 4(b). It is not possible to discriminate between the two mechanisms as, because of the weak polarization of the electron, they are both responsible of a small decrease of few percent of the circular polarization rate.

More interesting is the comparison with the data where a DNSP is created [red curve on the left panel of Fig. 7(b)]. In this later case, a fast decrease of the negative polarization rate is observed when increasing the transverse field from 0 to $10 \mathrm{mT}$. The half width at half maximum of the depolarization curve is $\approx 5 \mathrm{mT}$. This efficient depolarization of the resident electron is due to the precession of the coupled electron-nuclei system. ${ }^{14,20,34}$ After this fast depolarization of the electron, the negative polarization rate reaches the value observed in the absence of DNSP (i.e., under fast $\sigma+/ \sigma-$ modulated excitation, black curve). This is also observed in the modulation frequency dependence of the polarization rate presented in Fig. 4(a).

We observe that the depolarization curve in transverse magnetic field in the presence of nuclear spin polarization [Fig. 8(a)] strongly depends on the excitation power and deviates from a Lorentzian shape at high excitation power. For the later case, the negative polarization rate seems to be weakly affected by the first few milli-Tesla of transverse magnetic field, and then decreases abruptly.

The width of such depolarization curve is typically $50 \mathrm{mT}$ in a singly charged InAs/GaAs QDs in the presence of nuclear spin polarization. ${ }^{35}$ In InAs QDs, an influence of the magnetic anisotropy of the nuclei produced by the in-plane strain is also observed in the transverse magnetic field dependence of the Overhauser field. ${ }^{36,37}$ This cannot be the case in CdTe QDs as the nuclear spins $I=1 / 2$ for $\mathrm{Cd}$ and $\mathrm{Te}$.

The power dependence observed in our system could arise as the electron is pumped faster than the precession in the transverse applied $\left(\tau_{\text {press }}^{e}=4 \mathrm{~ns}\right.$ at $\left.B_{x}=5 \mathrm{mT}\right)$. At hight excitation intensity, the resident electron is replaced by an injected spin polarized electron faster than the precession in the transverse magnetic field. The depolarization of the electron spin is then given by the power dependent Hanle curve:

$$
S_{z}(\Omega)=\frac{S_{z}(0)}{\left[1+(\Omega \tau)^{2}\right]},
$$

where $\Omega=g_{e} \mu_{B} B / \hbar$ and $1 / \tau=1 / \tau_{p}+1 / \tau_{s}$ with $1 / \tau_{p}$ the pumping rate and $1 / \tau_{s}$ the electron spin relaxation rate. However, this power broadening does not explain the slight deviation from the Lorentzian shape we observed at high excitation intensity in Fig. 8(b).

The creation of DNSP could also be faster than the nuclei precession $\left(\tau_{\text {press }}^{N}=5 \mu \mathrm{s}\right.$ at $\left.B_{x}=5 \mathrm{mT}\right)$. The decrease of the electron polarization in a transverse magnetic field can then be influenced by the decrease of the steady state nuclear field. As a result of this decrease, the total in-plane component of the magnetic field which controls the electron precession increases more slowly than the external field $B_{x}$ : the precession of the electron would be efficiently blocked by the Overhauser field, and the electron polarization would be conserved. Such a scenario would be specific to II-VI quantum dots, where the build up of DNSP is fast enough to block the precession of nuclei. This point requires further investigation.

\section{B. Dynamics of the nuclear spin polarization}

In order to analyze quantitatively the buildup time and the characteristic amplitude of the polarization transient induced by the DNSP, we perform a time-resolved measurement using a $100 \mu$ s pulse of a $\sigma+$ helicity, followed by a $50 \mu$ s dark time during which the DNSP relaxes partially (quantitative analysis of this relaxation will be done in the next section and is indeed found to occur on a time scale shorter than $50 \mu \mathrm{s}$ for $B_{z}<5 \mathrm{mT}$ ). This experimental configuration enables us to fit the observed DNSP transient by an exponential variation, permitting to extract a characteristic rate $1 / \tau$ and amplitude $\Delta I$ [Fig. 9(a)]. 

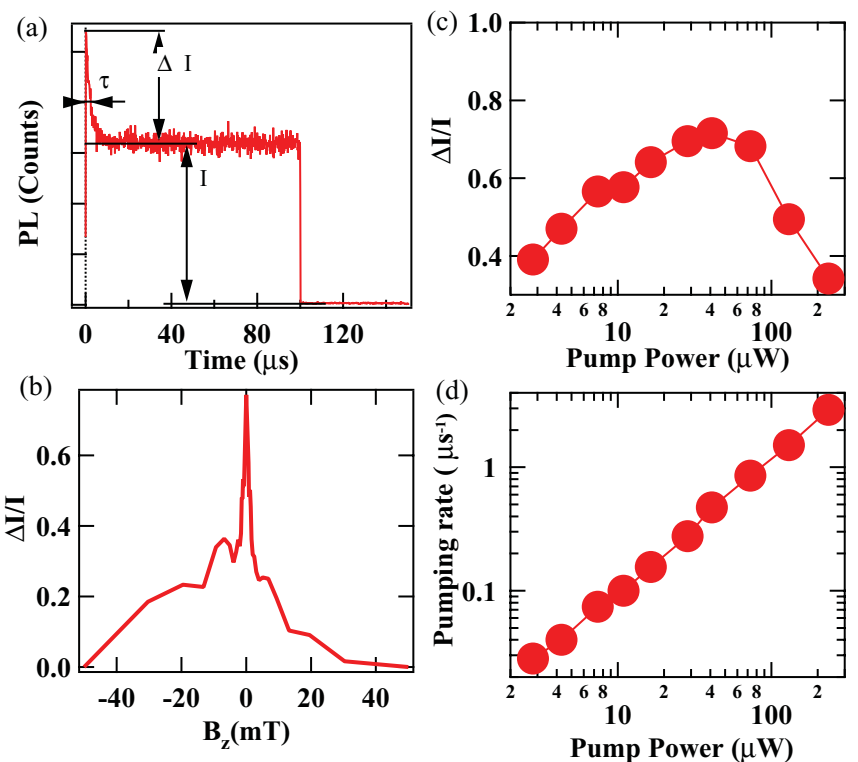

FIG. 9. (Color online) Variation of the amplitude (c) and characteristic time (d) of the DNSP transient under $\sigma+$ excitation [an example is presented in (a)] as a function of the excitation intensity at $B_{z}=0 \mathrm{mT}$ with a constant dark time $\tau_{\text {dark }}=50 \mu \mathrm{s}$. (b) Variation of the amplitude of the DNSP transient with a magnetic field applied along the QD growth axis.

We observe in Fig. 9(d) a linear increase of the pumping rate with the excitation power. While the build up of DNSP takes a few milliseconds in III-V materials at $B=0 \mathrm{~T},{ }^{10}$ it occurs in the microsecond range in our case. This results from the strong localization of the electron in II-VI quantum dots: the builtup rate of DNSP scales as ${ }^{38}\left|\Psi_{e}\right|^{4}$ so that we typically expect $\tau_{\mathrm{II}-\mathrm{VI}} / \tau_{\mathrm{III}-\mathrm{V}} \approx 8000^{2} /\left(10^{5}\right)^{2} \approx 5 \times 10^{-3}$.

The amplitude of the pumping transient $(\Delta I / I)$ presented in Fig. 9(c) increases linearly at low excitation power, reaches a maximum, and decreases at high power. The increase is attributed to an increase of the nuclear spin polarization. The reduction at high excitation power likely comes from a decoupling of the dynamics of the electron spin from the fluctuating nuclear spin. As we have already seen in Fig. 6(c), at high excitation intensity, optical pumping of the electron spin becomes faster than the precession in the fluctuating field of the nuclear spin $B_{f}$ and the measured polarization rate of the $X^{-}$becomes less sensitive to the polarization of the nuclei. A similar decrease of the amplitude of the transient is observed under a magnetic field of a few milli-Tesla applied along the QD growth axis.

The magnetic field dependence, of $\Delta I / I$ is shown in Fig. 9(b). We observe an important decrease of $\Delta I / I$ as soon as a few milli-Tesla are applied along the QD growth axis $z$. This fast decrease mainly comes from the increase of the relaxation time of the DNSP under magnetic field (this increase of the relaxation time is evidenced in Fig. 11 and will be further discussed): As the nuclear spin polarization does not fully relax during the dark time, the amplitude of the pumping transient decreases. This increase of the relaxation time explains the general shape at fields lower than a few milli-Tesla. At larger fields, a decrease also occurs when the static magnetic field exceed the fluctuating nuclear field. The
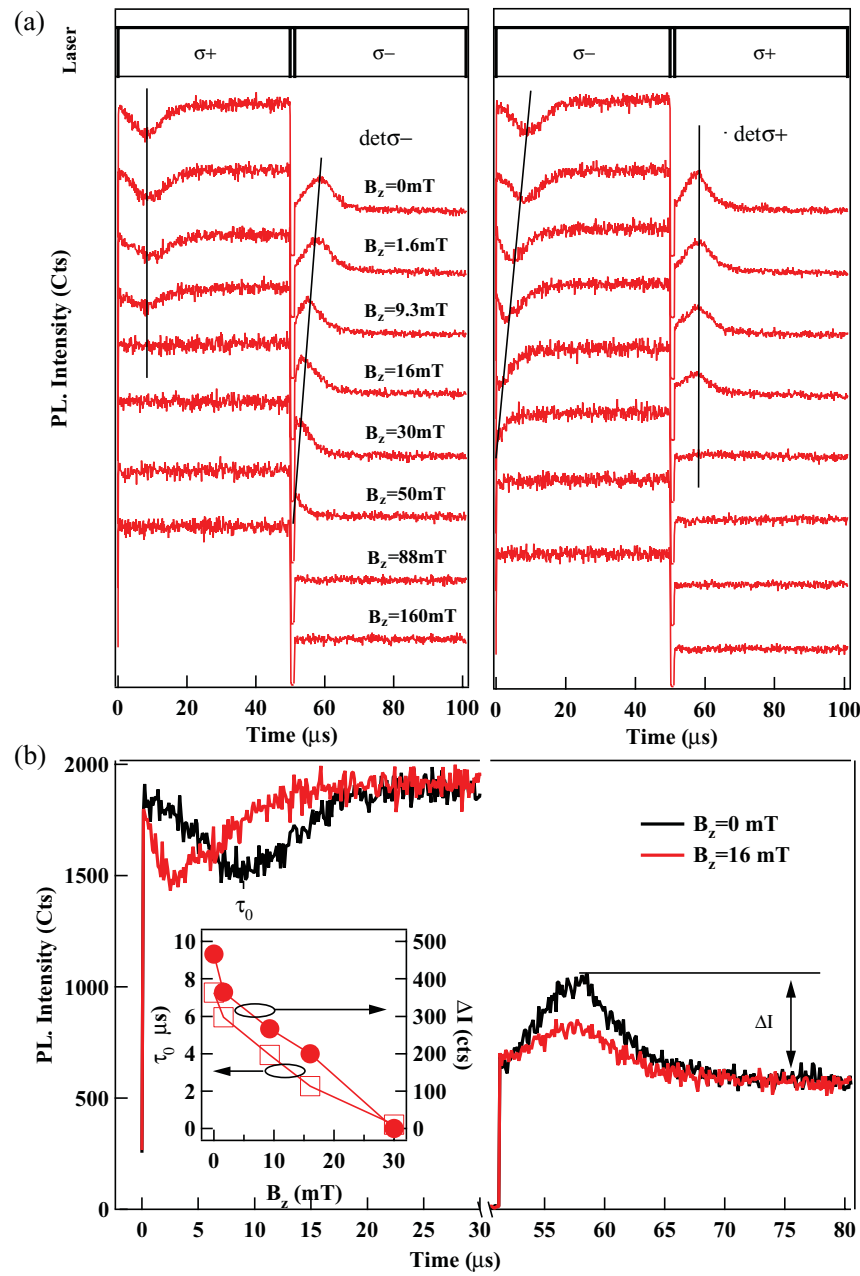

FIG. 10. (Color online) (a) Magnetic field dependence of the destruction and build up of the nuclear spin polarization observed under $\sigma+/ \sigma-$ modulated excitation (left: detection $\sigma-$, right: detection $\sigma+$ ). A detailed view of the transients is presented in (b). The inset shows the magnetic field dependence of the transient amplitude $(\Delta I)$ and position $\left(\tau_{0}\right)$.

magnetic field dependence of $\Delta I / I$ presents an asymmetry as the magnetic field is reversed. Similarly to the calculated asymmetry presented in Fig. 1 and the observed asymmetry presented in Fig. 7(a), this is the signature of the creation of an effective internal field with well defined direction. The faster drop of $\Delta I / I$ in a positive magnetic field comes from the increase of the influence of the fluctuating nuclear field $B_{f}$ when the external magnetic field compensates the Overhauser field. Such behavior has already been observed on ensemble of negatively charged CdSe/ZnSe QDs. ${ }^{4}$

Our study of the DNSP buildup time scales was complemented by adding a magnetic field in the Faraday configuration in the time-resolved pumping experiments. Under $\sigma+/ \sigma-$ modulated excitation, each switching of the polarization results in an instantaneous (on the time scale of the nuclear spin dynamics) change in the electron spin polarization followed by a slow evolution due to the repolarization of the nuclei. This repolarization process is responsible for the minimum observed in the time evolution of the negative polarization rate. Under a magnetic field, an asymmetry between the 

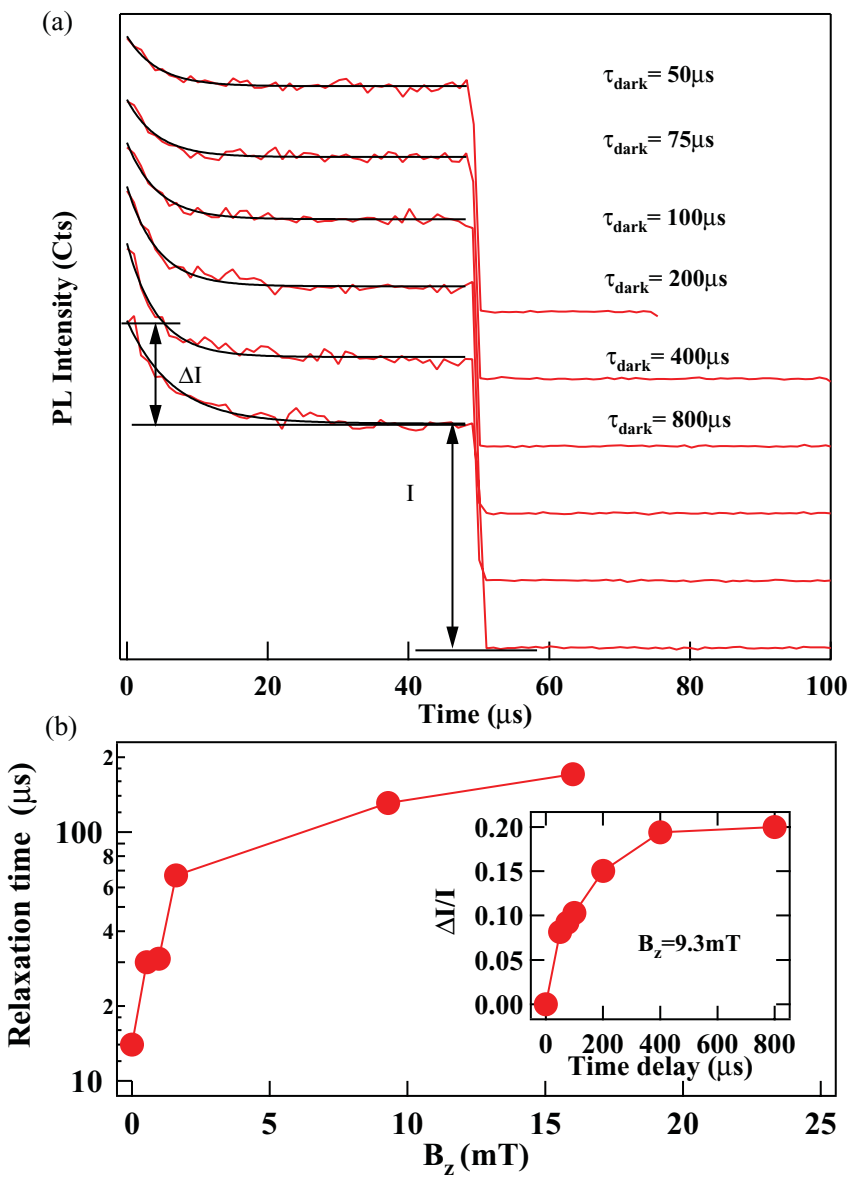

FIG. 11. (Color online) (a) Evolution of the DNSP transient, under a magnetic field $B_{z}=9.3 \mathrm{mT}$, with the variation of the dark time introduced between circularly polarized light trains of constant length. (b) Magnetic field dependence of the nuclear spin relaxation time. The inset show the evolution of amplitude of the DNSP transient with the dark time for $B_{z}=9.3 \mathrm{mT}$.

cases of $\sigma+$ and $\sigma-$ excitation is observed in the dynamics of the coupled electron-nuclei spin system (Fig. 10). Under $\sigma+$ excitation, as expected, the application of a magnetic field along $z$ progressively decreases the influence of the nuclear spin fluctuations on the electron-spin dynamics and the minimum in the electron polarization rate vanishes.

The behavior of the electron polarization is different under $\sigma-$ excitation: we observe an acceleration with the increase of $B_{z}$ of the destruction of the DNSP at the beginning of the $\sigma-$ pulse. This is illustrated in the inset of Fig. 11(b): The position of the minimum of polarization $\tau_{0}$ linearly shift from $\tau_{0} \approx 8 \mu$ s at $B_{z}=0 \mathrm{mT}$ to $\tau_{0} \approx 0 \mu \mathrm{s}$ at $B_{z} \approx 30 \mathrm{mT}$.

At the end of the $\sigma+$ light train, the polarized light has created a nuclear field $B_{N}^{\sigma+}$ antiparallel to $B_{z}$. At some time after switching to $\sigma-$ excitation, $B_{N}$ decreases and approaches $-B_{z}$. At this point, the nonlinear feedback process in the electron-nuclei "flip-flops" starts and accelerates the depolarization until the DNSP vanishes. Simultaneously, the absolute value of the negative polarization reaches a minimum. Then the nuclei are repolarized by the $\sigma-$ excitation until $B_{N}$ reaches $B_{N}^{\sigma-}$ parallel to $B_{z}$. Consequently, as observed in Fig. 10(b), under $\sigma$ - excitation the destruction of the DNSP is expected to be faster than its buildup. However, it is not clear why such a magnetic field dependent acceleration is not observed during the build up of the DNSP at the end of the transient in $\sigma+$ polarization when $B_{N}^{\sigma+}$ reaches $-B_{z}$. To fully understand this behavior, a complete model of the coherent dynamics of coupled electron and nuclear spins in a weak Faraday magnetic field should be developed. Such model at zero field has already shown that the minimum of $\left\langle S_{z}\right\rangle$ can apparently be shifted from the point $\left\langle I_{z}\right\rangle=0 .{ }^{32}$

\section{Nuclear spin polarization decay}

In order to investigate the variation with magnetic field of the relaxation time in the dark of the DNSP, we follow the protocol shown in Fig. 11. For a given magnetic field, we prepare a DNSP and measure after a time $\tau_{\text {dark }}$ the amplitude of the transient, corresponding to the partial relaxation of the nuclear polarization. As $\tau_{\text {dark }}$ is increased, this amplitude saturates, demonstrating the full relaxation. The variation of the amplitude of the transient with $\tau_{\text {dark }}$ is used to estimate the relaxation time of the DNSP at a given magnetic field.

The evolution of this relaxation time is presented in Fig. 11(b). It ranges from $14 \mu \mathrm{s}$ at $B=0 \mathrm{~T}$ to $170 \mu \mathrm{s}$ at $B=$ $16 \mathrm{mT}$. The relaxation rate is one order of magnitude faster than the one observed by Feng et al. on ensemble of CdSe/ZnSe QDs, ${ }^{3}$ and the one expected from nuclear dipole-dipole interactions. Furthermore, in InAs/GaAs Schottky structure the decay in electron charged dots occurred in a millisecond time scale ${ }^{10}$ while nuclear spin lifetime in an empty dot has been shown to exceed $1 \mathrm{~h} .{ }^{39}$

The magnetic field dependence of the DNSP relaxation presents a significant increase of the decay time over the first few milli-Tesla. It has been demonstrated that a magnetic field of $1 \mathrm{mT}$ efficiently inhibits nuclear dipole-dipole interactions in III-V materials. ${ }^{10}$ Since this interaction is expected to be smaller in our system with diluted nuclear spins, we can definitely rule out dipole-dipole interaction as a major cause of DNSP relaxation.

Cotunneling to the close-by reservoir could be responsible for this depolarization. Via hyperfine-mediated flip-flop, the randomization of the electron spin creates an efficient relaxation of the nuclei. Following Merkulov et al. ${ }^{38}$ this relaxation time is given by

$$
T_{1 e}^{-1}=\frac{2\left\langle\omega^{2}\right\rangle\|s\|^{2} \tau_{c}}{3\left[1+\left(\Omega \tau_{e}\right)^{2}\right]} .
$$

In this expression $\omega$ is the precession frequency of the nuclei in the Knight field, $\tau_{e}$ is the correlation time of the electron (in the dark), and $\Omega$ is the precession frequency of the electron in the Overhauser field. At last, $\|s\|^{2}$ is equal to $s(s+1)=3 / 4$. The fastest relaxation we expect from this process can be estimated taking $\Omega=\Omega_{\text {fluc }}=2 \pi / 2 \mathrm{~ns}^{-1}$ and $\tau_{c}=10 \mathrm{~ns}$. We obtain $T_{1 e} \approx 200 \mu$ s which is not fast enough. Therefore, we are tempted to conclude that cotunneling alone cannot explain the observed dynamics.

Another mechanism to consider is the depolarization resulting from an electron-mediated nuclear dipole-dipole interaction. This results in exchange constants between the nuclei which typically scale as $A^{2} /\left(N^{2} \epsilon_{z}\right)$. The resulting rate of nuclear-spin depolarization is $T_{\text {ind }}^{-1} \approx A^{2} /\left(N^{3 / 2} \hbar \epsilon_{z}\right)$, where 
$\epsilon_{z}$ is the Zeeman splitting of the electron. This mechanism could explain a depolarization of the nuclei on a microsecond scale. ${ }^{40,41}$ However, this expression gives only a minor bound to the relaxation time because the inhomogeneity of the Knight field can strongly inhibit this decay. ${ }^{42,43}$ A magnetic field along the $z$ axis is expected to affect this process, progressively decoupling the nuclei from the indirect coupling created by the electron, as observed in our experiments in the first few milli-Tesla (Fig. 11). The electron-induced nuclear depolarization was demonstrated in Ref. 10 in which the millisecond relaxation was completely suppressed using a voltage pulse on a Schottky diode in order to remove the resident electron.

\section{CONCLUSION}

In summary we have evidenced in the PLE spectra of a negatively charged CdTe QD the polarized fine structure of the triplet states of the charged exciton. We have studied, using PL decay measurements, the dynamics of the injection of spin polarized photocarriers as a function of the energy of the injection. We have shown that the injection above the triplet states of the charged exciton can be use to pump the resident electron on a time scale of 10-100 ns and to create a dynamic nuclear spin polarization. At $B=0 \mathrm{~T}$, the creation of the dynamic nuclear spin polarization can be as fast as a few microseconds, and the decay of the nuclear polarization, attributed to an electron mediated relaxation, is $\approx 10 \mu \mathrm{s}$. The measured dynamics are $\approx 10^{3}$ faster than the ones observed in III-V QDs at $B=0 \mathrm{~T}$. The relaxation time of the coupled electron-nuclei system is increased by one order of magnitude under a magnetic field of $5 \mathrm{mT}$. The magnetic-field dependence of the PL polarization rate revealed that the nuclear spin fluctuations are the dominant process in the dephasing of the resident electron. We proved that this dephasing is efficiently suppressed by a large dynamic nuclear spin polarization at $B=0 \mathrm{~T}$.

\section{ACKNOWLEDGMENTS}

This work is supported by the French ANR contract QuAMOS and EU ITN project Spin-Optronics. *lucien.besombes@grenoble.cnrs.fr

${ }^{1}$ A. Khaetskii, D. Loss, and L. Glazman, Phys. Rev. B 67, 195329 (2003).

${ }^{2}$ W. K. Liu, K. M. Whitaker, A. L. Smith, K. R. Kittilstved, B. H. Robinson, and D. R. Gamelin, Phys. Rev. Lett. 98, 186804 (2007). ${ }^{3}$ D. H. Feng, I. A. Akimov, and F. Henneberger, Phys. Rev. Lett. 99, 036604 (2007).

${ }^{4}$ I. A. Akimov, D. H. Feng, and F. Henneberger, Phys. Rev. Lett. 97, 056602 (2006).

${ }^{5}$ B. Eble, O. Krebs, A. Lemaitre, K. Kowalik, A. Kudelski, P. Voisin, B. Urbaszek, X. Marie, and T. Amand, Phys. Rev. B 74, 081306(R) (2006).

${ }^{6}$ P. Wojnar, C. Bougerol, E. Bellet-Amalric, L. Besombes, H. Mariette, and H. Boukari, J. Cryst. Growth 335, 28 (2011).

${ }^{7}$ C. Le Gall, R. S. Kolodka, C. L. Cao, H. Boukari, H. Mariette, J. Fernández-Rossier, and L. Besombes, Phys. Rev. B 81, 245315 (2010).

${ }^{8}$ D. Paget, G. Lampel, B. Sapoval, and V. I. Safarov, Phys. Rev. B 15, 5780 (1977).

${ }^{9}$ C. Testelin, B. Eble, F. Bernardot, G. Karczewski, and M. Chamarro, Phys. Rev. B 77, 235306 (2008).

${ }^{10}$ P. Maletinsky, C. W. Lai, A. Badolato, and A. Imamoglu, Phys. Rev. B 75, 035409 (2007).

${ }^{11}$ L. Besombes, L. Marsal, K. Kheng, T. Charvolin, Le Si Dang, A. Wasiela, and H. Mariette, J. Cryst. Growth 214-215, 742 (2000).

${ }^{12}$ Y. Leger, L. Besombes, L. Maingault, and H. Mariette, Phys. Rev. B 76, 045331 (2007).

${ }^{13}$ D. E. Gray, Ed. American Institute of Physics Handbook, 3rd ed. (McGraw-Hill, New York, 1972).

${ }^{14} \mathrm{~F}$. Meier and B. P. Zakharchenya, Optical Orientation (Elsevier, Amsterdam, 1984).

${ }^{15}$ M. N. Makhonin, E. A. Chekhovich, P. Senellart, A. Lemaitre, M. S. Skolnick, and A. I. Tartakovskii, Phys. Rev. B 82, 161309 (2010).

${ }^{16}$ C. W. Lai, P. Maletinsky, A. Badolato, and A. Imamoglu, Phys. Rev. Lett. 96, 167403 (2006).
${ }^{17}$ A. Abragam, Principles of Nuclear Magnetism (Oxford University Press, Oxford, 1961), Chap. 8.

${ }^{18}$ A. I. Tartakovskii, T. Wright, A. Russell, V. I. Falko, A. B. Vankov, J. Skiba-Szymanska, I. Drouzas, R. S. Kolodka, M. S. Skolnick, P. W. Fry, A. Tahraoui, H.-Y. Liu, and M. Hopkinson, Phys. Rev. Lett. 98, 026806 (2007).

${ }^{19}$ M. I. Dyakonov, Spin Physics in Semiconductors (Springer, Berlin, 2008).

${ }^{20}$ R. V. Cherbunin, S. Yu. Verbin, T. Auer, D. R. Yakovlev, D. Reuter, A. D. Wieck, I. Ya. Gerlovin, I. V. Ignatiev, D. V. Vishnevsky, and M. Bayer, Phys. Rev. B 80, 035326 (2009).

${ }^{21}$ D. Gammon, Al. L. Efros, T. A. Kennedy, M. Rosen, D. S. Katzer, D. Park, S. W. Brown, V. L. Korenev, and I. A. Merkulov, Phys. Rev. Lett. 86, 5176 (2001).

${ }^{22}$ M. E. Ware, E. A. Stinaff, D. Gammon, M. F. Doty, A. S. Bracker, D. Gershoni, V. L. Korenev, S. C. Badescu, Y. Lyanda-Geller, and T. L. Reinecke, Phys. Rev. Lett. 95, 177403 (2005).

${ }^{23}$ S. Laurent, M. Senes, O. Krebs, V. K. Kalevich, B. Urbaszek, X. Marie, T. Amand, and P. Voisin, Phys. Rev. B 73, 235302 (2006).

${ }^{24}$ M. Ikezawa, B. Pal, Y. Masumoto, I. V. Ignatiev, S. Yu. Verbin, and Ilya Ya. Gerlovin, Phys. Rev. B 72, 153302 (2005).

${ }^{25}$ A. S. Bracker, E. A. Stinaff, D. Gammon, M. E. Ware, J. G. Tischler, A. Shabaev, Al. L. Efros, D. Park, D. Gershoni, V. L. Korenev, and I. A. Merkulov, Phys. Rev. Lett. 94, 047402 (2005).

${ }^{26}$ A. Shabaev, E. A. Stinaff, A. S. Bracker, D. Gammon, A. L. Efros, V. L. Korenev, and I. Merkulov, Phys. Rev. B 79, 035322 (2009).

${ }^{27}$ T. Flissikowski, I. A. Akimov, A. Hundt, and F. Henneberger, Phys. Rev. B 68, 161309(R) (2003).

${ }^{28}$ S. Cortez, O. Krebs, S. Laurent, M. Senes, X. Marie, P. Voisin, R. Ferreira, G. Bastard, J-M. Gerard, and T. Amand, Phys. Rev. Lett. 89, 207401 (2002).

${ }^{29}$ E. S. Moskalenko, L. A. Larsson, and P. O. Holtz, Phys. Rev. B 80, 193413 (2009).

${ }^{30}$ I. A. Merkulov, Al. L. Efros, and M. Rosen, Phys. Rev. B 65, 205309 (2002). 
${ }^{31}$ M. Y. Petrov, I. V. Ignatiev, S. V. Poltavtsev, A. Greilich, A. Bauschulte, D. R. Yakovlev, and M. Bayer, Phys. Rev. B 78, 045315 (2008).

${ }^{32}$ M. Y. Petrov, G. G. Kozlov, I. V. Ignatiev, R. V. Cherbunin, D. R. Yakovlev, and M. Bayer, Phys. Rev. B 80, 125318 (2009).

${ }^{33}$ X. M. Dou, B. Q. Sun, D. S. Jiang, H. Q. Ni, and Z. C. Niu, Europhys. Lett. 98, 17007 (2012)

${ }^{34}$ R. Oulton, A. Greilich, S. Yu. Verbin, R. V. Cherbunin, T. Auer, D. R. Yakovlev, M. Bayer, I. A. Merkulov, V. Stavarache, D. Reuter, and A. D. Wieck, Phys. Rev. Lett. 98, 107401 (2007).

${ }^{35}$ P. Desfonds, B. Eble, F. Fras, C. Testelin, F. Bernardot, M. Chamarro, B. Urbaszek, T. Amand, X. Marie, J. M. Grard, V. Thierry-Mieg, A. Miard, and A. Lematre, Appl. Phys. Lett. 96, 172108 (2010).

${ }^{36}$ R. I. Dzhioev and V. L. Korenev, Phys. Rev. Lett. 99, 037401 (2007).
${ }^{37}$ O. Krebs, P. Maletinsky, T. Amand, B. Urbaszek, A. Lemaitre, P. Voisin, X. Marie, and A. Imamoglu, Phys. Rev. Lett. 104, 056603 (2010).

${ }^{38}$ I. A. Merkulov, G. Alvarez, D. R. Yakovlev, and T. C. Schulthess, Phys. Rev. B 81, 115107 (2010).

${ }^{39}$ P. Maletinsky, M. Kroner, and A. Imamoglu, Nat. Phys. 5, 407 (2009).

${ }^{40}$ D. Klauser, W. A. Coish, and D. Loss, Phys. Rev. B 78, 205301 (2008).

${ }^{41}$ D. Klauser, W. A. Coish, and D. Loss, Phys. Rev. B 73, 205302 (2006).

${ }^{42}$ C. Deng and X. Hu, Phys. Rev. B 72, 165333 (2005).

${ }^{43}$ E. A. Chekhovich, M. N. Makhonin, J. Skiba-Szymanska, A. B. Krysa, V. D. Kulakovskii, M. S. Skolnick, and A. I. Tartakovskii, Phys. Rev. B 81, 245308 (2010). 